\title{
ON THE EXISTENCE OF INITIAL MODELS FOR PARTIAL (HIGHER-ORDER) CONDITIONAL SPECIFICATIONS
}

\author{
Egidio Astesiano and Maura Cerioli \\ Dipartimento di Matematica - Università di Genova \\ Via L.B. Alberti 4 - 16132 Genova Italy
}

\begin{abstract}
Partial higher-order conditional specifications may not admit initial models, because of the requirement of extensionality, even when the axioms are positive conditional. The main aim of the paper is to investigate in full this phenomenon.

If we are interested in term-generated initial models, then partial higher-order specifications can be seen as special cases of partial conditional specifications, i.e. specifications with axioms of the form $\wedge \Delta \supset \mathcal{E}$, where $\Delta$ is a denumerable set of equalities, $\varepsilon$ is an equality and equalities can be either strong or existential. Thus we first study the existence of initial models for partial conditional specifications.

The first result establishes that a necessary and sufficient condition for the existence of an initial model is the emptiness of a certain set of closed conditional formulae, which we call "naughty". These naughty formulae can be characterized w.r.t. a generic inference system complete w.r.t. closed existential equalities and the above condition amounts to the impossibility of deducing those formulae within such a system. Then we exhibit an inference system which we show to be complete w.r.t closed equalities; the initial model exists if and only if no naughty formula is derivable within this system and, when it exists, can be characterized, as usual, by the congruence associated with the system.

Finally, applying our general results to the case of higher-order specifications with positive conditional axioms, we obtain necessary and sufficient conditions for the existence of term-generated initial models in that case.
\end{abstract}

\section{An overview}

Partial algebraic specifications ([BW1, R, WB]) are one of the most interesting specification paradigms. Originally proposed as a support to the stepwise refinement procedure, they have found more recently interesting applications to the specification of concurrency and of formal semantics of languages (see eg [BW2, AR1, AR2]).

Higher-order specifications creep in naturally in the specification activity because of the application requirements (see [AR1]) and of methodological considerations (see eg [M, MTW]).

Unfortunately higher-order specifications present a rather different situation from the first-order case, due to the extensionality requirement. The most striking feature is that the existence of initial model is not guaranteed, even if the axioms are in a form which looks a natural extension of the one that guarantees the existence in the first-order case. Let us consider this problem in some more detail.

A classical result [BW1] states that the initial partial model exists (and is term-generated) in the class of all partial models, if the specification is positive conditional, i.c. the axioms have the form

$$
\wedge_{i=1, \ldots, n} t_{i}=e_{i}^{\prime} \supset t=t^{\prime}
$$

where $=\mathrm{e}$ and $=$ denote respectively existential (the sides are both defined and equal) and strong (the sides are either existentially equal or are both undefined) equality.

This result can be generalized in two directions: in the axioms the conjunction may be infinitary and the consequence may be an inequality; this situation is also the most general guaranteeing the existence of the initial term-generated model (see $[T]$ for references and the most general results in this direction).

This work has been partly supported by CNR-Italy (Progetto Strategico "Software: ricerche di base e applicazione") and 
Consider now the case of higher-order specifications, where starting from some basic sorts we have inductively functional sorts of the form $s_{1} \times \ldots \times s_{n} \rightarrow s$, the corresponding carriers in a model are required to be sets of partial functions and the axioms may of course contain equalities between terms of functional sort. It is quite instructive to analyse a simple example, the specification $\mathrm{T}_{0}$ :

basic sorts: $s$

operation symbols: $\mathrm{e}: \rightarrow \mathrm{s} \quad \mathrm{f}, \mathrm{g}: \mathrm{s} \rightarrow \mathrm{s}$

functional sorts: $s \rightarrow s$

axioms: $\quad D(f), D(g), f=e g \supset D(f(e))$

where we use definedness predicate $D$, and $D(t)$ is equivalent to $t=e t$.

Recall now that the term generated initial model $I$ of $T_{0}$ (and of any partial specification), if it exists, is such that an existential equality holds in I iff it holds in any model; in particular I is minimally defined: a term is defined in I iff it is defined in every model.

Since clearly there exist models of $T_{0}$ where $f(e)$ is undefined and the same happens for $g(e)$, both $f(e)$ and $g(e)$ should be undefined and hence equal in I. By partiality and extensionality, I being term-generated, we should conclude that $f=g$ holds in $I$ and this clearly contradicts $I$ being a model, since from the axioms we should conclude that $f(e)$ is defined in $I$. It is not difficult to understand that all the trouble comes from the fact that extensionality amounts to requiring that the term-generated models satisfy the supplementary axiom

$$
\wedge_{t \in W_{\Sigma}} f(t)=g(t) \supset f=g
$$

where in the premise the equality is strong and this makes the axiom essentially non-positive conditional (essentially, since neither the definedness of $f(t)$ nor of $g(t)$ can be logically derived).

Notice also that $T_{0}$ does not admit an initial model tout-court, even if we drop the condition about being term-generated (see Remark in sec. 4).

The $T_{0}$ example suggests that the anomaly shown is not peculiar of higher-order specifications but is typical instead of conditional specifications, i.e. specifications where the axioms have the form $\wedge \Delta \supset \varepsilon$, where $\Delta$ is a set of possibly strong equalities and $\varepsilon$ is an equality.

Then if we confine ourselves to consider term-extensional models, i.e. models where two functions are equal if (and only if) they are equal on all term-generated arguments, we can express the (term-)extensionality axioms in the form

where $\bar{t}=\left(t_{1}, \ldots, t_{n}\right)$

$$
\wedge \bar{t} \in W_{\Sigma} f(\bar{t})=g(\bar{t}) \supset f=g
$$

which is conditional. Moreover higher-order specifications can be reduced to first-order specifications (see, e.g. [MTW]) by using the apply functions, so that a term $f(t)$ is an abbreviation of apply(f,t). Thus we are led to consider the mentioned initiality problem in the (more general) framework of partial first-order conditional specifications, with axioms of the form $\wedge \Delta \supset \varepsilon$, where $\Delta$ is a denumerable set of equalities (not necessarily existential) and $\varepsilon$ is an equality, and of course sorts are now only sorts of individual elements.

We present in this paper a complete answer to the initiality problem for partial conditional specifications, giving necessary and sufficient conditions for the existence of the initial model (and as usual characterizing that model, if it exists, as the quotient algebra w.r.t. the congruence associated with an inference system which is complete w.r.t. closed equalities). Since the detailed mathematical treatment is technically rather difficult, we explain briefly the main underlying ideas, with the help of some examples.

Anomalies similar to the one shown by the example. $T_{0}$ can be seen also in the following two examples (discussed in a more technical context at the end of sec. 1):

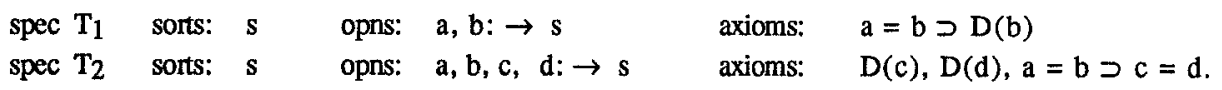

The lack of the initial model for $T_{1}$ is due to the particular form of the axiom $a=b \supset D(b)$ : since there exist models where $a$ is undefined and the same happens for $b$, if the initial model $I$ exists, then $a^{I}$ and $b^{I}$ are both undefined and equal, so that the axiom, which would imply $D(b)$, cannot be met. A partly similar situation is shown by $T_{2}$; the combination of the three axioms $D(c), D(d)$ and $a=b \supset c=d$ produces a situation analogous to the one just discussed: if the initial model I exists, then not only $\mathrm{a}^{\mathrm{I}}$ and $\mathrm{b}^{\mathrm{I}}$ would be undefined and equal, but also $c^{\mathrm{I}}$ and $d^{I}$ should be different, otherwise $c^{I}=b^{I}$, being an existential equality, should hold in every model what is patently false; hence the axiom $a=b \supset c=d$, which would imply $c=d$, cannot be met. Note that the axiom $a=b \supset c=d$ in itself does not prevent the existence of an initial model (see remarks at the end of Sec. 1). Analogously the formula $a=b \supset D(b)$ of $T_{1}$ would not create any problem if we could deduce from the axioms, 
say, that $D(a)$ holds in every model, which would imply that $a=b \supset D(b)$ is logically equivalent to a positive conditional axiom.

Hence formulae like the two ones above are causing trouble in connection with what we can logically deduce from the axioms (equivalently, what is true in every model); consequently we call them "naughty" w.r.t. the specification. The first main result of the paper consists indeed in capturing precisely and formalizing the notion of "naughty formula" for a specification and establishing (theorem 1.6) that the initial model exists if and only if the set of naughty formulae is empty. Then naughty formulae can be characterized as a particular subset of the conditional formulae deducible within any inference system complete w.r.t. closed existential equalities, and so the initial model exists iff we cannot derive any of these naughty formulae in such a system. (second main result, theorem 2.8 ). All the above motivates our third main result showing an inference system complete w.r.t. closed (strong and existential) equalities. First of all note that since the axioms may be infinitary (and this is essential, since we want to handle also the term-extensionality axioms), the inference system is within infinitary logic (where no general completeness result is available as for first-order theories). Moreover our aim has been to obtain a system which reduces, for positive conditional specifications, to the one naturally associated with positive conditional axioms; and indeed we add to that system only one inference rule which is rather intuitive in the finitary case. Let us consider a finitary significant example.

Consider the specification $\mathrm{T}_{4}$ defined by

sort: $s$ opns: $a, b, c: \rightarrow s$ axioms: $\quad D(a) \supset a=b, D(b) \supset a=b, a=b \supset D(c)$.

It is not difficult to show that $T_{4}$ admits an initial model $\mathrm{I}$, consisting of a one point carrier containing just the value of $c$, with $a$ and $b$ undefined. Moreover $a=b$ and $D(c)$ hold in every model.

However the usual inference system which is complete for positive conditional axioms (see def. 2.2 for an infinitary version) is not powerful enough to deduce $a=b$ nor $D(c)$ and hence the quotient algebra w.r.t. the congruence associated with the system is not a model.

This limitation disappears if we can apply the following clearly sound rule (see Sec. 3).

*

$$
\frac{\wedge\left(\Theta_{1} \cup\{D(t)\}\right) \geq \alpha, \wedge\left(\Theta_{2} \cup\left[D\left(t^{\prime}\right)\right\}\right) \supset \alpha, \wedge\left(\Theta_{3} \cup\left\{t=t^{\prime}\right)\right) \geq \alpha}{\wedge\left(\Theta_{1} \cup \Theta_{2} \cup \Theta_{3}\right) \supset \alpha}
$$

where $\Theta_{1}, \Theta_{2}, \Theta_{3}$ are finite sets of closed equations and definedness assertions, $\alpha$ is either a closed equation or a closed definedness assertion and $t, t^{\prime}$ are closed terms of the same sort.

Indeed recalling that $a=b \supset a=b$ and instantiating * we can deduce $a=b$ and hence $D(c)$. Our complete system adds to the usual conditional system just an infinitary version of rule * (see Def. 3.1); please note that, in order to handle the extensionality axiom which is infinitary, then we need to work within infinitary logic.

The first two main results completely settle the initiality question for conditional specifications. We can now apply these results to positive conditional higher-order specifications, accordingly to our previous discussion. Restricting ourselves to consider term-extensional models, the extensionality axiom is just an infinitary conditional axiom, the only one non-positive (for a positive conditional higher-order specification). Thus we get our fourth main result (theorem 4.5) which gives interesting necessary and sufficient conditions for the existence of the (term-generated) initial model.

The paper is organized as follows. The basic elementary definition about partial algebras are collected in appendix. In the first section we present the basic properties of conditional specifications, introduce the notion of naughty formula and give the first main result. In the second, after defining conditional inference systems and presenting one of them, we characterize the naughty formulae w.r.t. a conditional system and state the second main result. The third section introduces the extra rule to be added to the conditional system of sec. 2 and proves in outline the completeness w.r.t. closed equalities of the resulting system. Finally the fourth and last section applies the results to higher-order specifications and contains our fourth main result.

Due to lack of room, with the exception of an outline of the proof of the completeness result of sec. 3 , the proofs are omitted and will be found in a full version of this paper ([AC1]) more generally dealing with the existence of free objects and full equational deduction, generalizing and obtaining as a special case the corresponding MeseguerGoguen [MG] completeness result for total algebras. 


\section{Conditional partial specifications and initial models}

In the following we assume some familiarity with partial algebras (see [BW1, R, B]); however all the relevant definitions, concepts and results needed here are reported in appendix.

Def. 1.1. Let $\Sigma=(S, F)$ be a signature and $X$ be a family of S-sorted variables.

- The set $\mathrm{EForm}(\Sigma, \mathrm{X})$ of elementary formulae over $\Sigma$ and $\mathrm{X}$ is the set

$\left\{D(t) \mid t \in W_{\Sigma}(X)_{\mid s}\right\} \cup\left\{t=t^{\prime} \mid t, t^{\prime} \in W_{\Sigma}(X)_{\mid s}\right\}$,

where $\mathrm{D}$ denotes the definedness predicate (one for each sort; but sorts are omitted).

Note that $D(t)$ can be equivalently expressed by $t=e t$, where $=e$ denotes existential equality: $=e$ holds iff both sides are defined and equal; hence elementary formulae are just equalities either strong or existential.

- The set WForm $(\Sigma, X)$ of conditional formulae is the set

$\{\wedge \Delta \supset \varepsilon \mid \Delta \subseteq E F o r m(\Sigma, X), \Delta$ is countable, $\varepsilon \in \operatorname{EForm}(\Sigma, X)\}$.

If $\Delta$ is the empty set, then $\wedge \Delta \supset \mathcal{E}$ is an equivalent notation for the elementary formula $\varepsilon$. ( $\wedge \Delta$ is a notation for the couple $(\wedge, \Delta)$; see $[\mathrm{K}])$

- For every formula $\varphi$ let $\operatorname{Var}(\varphi)$ denote the set of all variables which appear in $\varphi$. A formula $\varphi$ is called closed iff $\operatorname{Var}(\varphi)$ is empty.

- A positive conditional formula is a conditional formula $\wedge \Delta \supset \varepsilon$ s.t. for every $t=t^{\prime}$ belonging to $\Delta$ either $\mathrm{D}(\mathrm{t})$ or $\mathrm{D}\left(\mathrm{t}^{\prime}\right)$ belongs to $\Delta$.

- If $A$ is a partial algebra, $\varphi$ is a formula and $V$ is a valuation for $\operatorname{Var}(\varphi)$ in $A$, then we say that $\varphi$ holds for $V$ in $A$ (equivalently: is satisfied for $V$ by $A$ ) and write $A \vDash V \varphi$ accordingly to the following definitions.

- $A \vDash V D(t)$ iff $t^{A, V}$ is defined;

- $A \vDash V t=t^{\prime}$ iff $t^{A}, V$ and $t^{\prime} A, V$ are either both defined and equal or both undefined;

- $A \vDash_{V} \wedge \Delta \supset \varepsilon$ iff $A \vDash_{V} \delta$ for all $\delta \in \Delta$ implies that $A \vDash V \varepsilon$.

We write $A \vDash \varphi$ for a formula $\varphi$ and say that $\varphi$ holds in (equivalently: is satisfied by, is valid in ) $A$ iff $A \vDash V \varphi$ for all valuation $V$ for $\operatorname{Var}(\varphi)$ in $A$.

In the following a generic elementary formula will be denoted by $\varepsilon$ or $\eta$ or $\gamma$ or $\delta$, while a generic conditional formula will be denoted by $\varphi$ or $\vartheta$ or $\psi$; moreover for all conditional formulae $\varphi=(\wedge \Delta \supset \varepsilon)$ we denote $\Delta$ by prem $(\varphi)$ and $\varepsilon$ by cons $(\varphi)$; finally we will use some equivalent notations:

$\wedge \Delta_{1} \wedge \ldots \wedge \wedge \Delta_{n} \supset \varepsilon$ is the same as $\wedge\left(\cup_{i=1} \ldots n \Delta_{i}\right) \supset \varepsilon$;

$\varepsilon_{1} \wedge \ldots \wedge \varepsilon_{n} \supset \varepsilon$ is the same as $\wedge\left\{\varepsilon_{1}, \ldots, \varepsilon_{n}\right\} \supset \varepsilon$

where $\Delta_{1}, \ldots, \Delta_{\mathrm{n}}$ are sets of elementary formulae and $\varepsilon_{1}, \ldots, \varepsilon_{\mathrm{n}}, \varepsilon$ are elementary formulae.

Def. 1.2 .

- A conditional type (also called conditional specification) consists of a signature $\Sigma$ and of a set Ax of conditional formulae over $\Sigma$. A generic conditional type will be denoted by $T$; the formulae belonging to $A x$ are called usually the axioms of $\mathrm{T}$ and are denoted by $\alpha$.

- A positive conditional type is a conditional type s.t. all its axioms are positive conditional formulae; a generic positive conditional type will be usually denoted by $\mathrm{PT}$.

- For every conditional type $T=(\Sigma, A x), \operatorname{PMod}(T)$ denotes the class of all models of $T$, ie the class of $\Sigma$-algebras satisfying every formula of $A x$, ie $\operatorname{PMod}(T)=\{A \mid A \in P A(\Sigma), A \vDash \alpha, \forall \alpha \in A x\}$.

- For every conditional type $T=(\Sigma, A x), P G e n(T)$ denotes the class of all term-generated models of $T$, ie $\operatorname{PGen}(\mathrm{T})=\operatorname{Gen}(\operatorname{PMod}(\mathrm{T}))$.

Prop. 1.3. If $C$ is a non-empty subclass of $\operatorname{PMod}(T)$ and either $T$ is a positive conditional type, or $C=\operatorname{MDef}(C)$, then $\mathrm{W}_{\Sigma} / \mathrm{K}^{C}$ is a model of $\mathrm{T}$.

\section{Theorem 1.4 .}

1) If $\mathrm{PT}$ is a positive conditional type, then $\mathrm{W}_{\Sigma} / \mathrm{K}^{\mathrm{PMod}}(\mathrm{PT})$ is initial in $\mathrm{PMod}(\mathrm{PT})$.

2) If $T$ is a conditional type s.t. $\operatorname{MDef}(\operatorname{PGen}(T))$ is not empty, then $W_{\Sigma} / K^{M D e f(P G e n(T)) ~ i s ~ i n i t i a l ~ i n ~}$ MDef(PGen(T)). 
3) A model $I$ is initial in PMod(T) iff it is initial in PGen(T).

4) If $I$ is initial in $\operatorname{PMod}(T)$, then $I \in \operatorname{MDef}(\operatorname{PGen}(T))$.

\section{Remarks.}

Conditional types are rather pathological w.r.t. positive conditional types. Remarks 1 and 2 below show the key features of this pathological behaviour. In particular the remark 3 shows the intrinsic irreducibility of conditional types to positive conditional types, even in the case when an initial model exists. remark 4 from same pathological examples extracts the essential idea for a solution of the problem of initiality, which is presented in the next section.

1. If $\mathrm{T}$ is a conditional type, then $\mathrm{MDef}(\mathrm{PGen}(\mathrm{T}))$ may be empty and thun there may not be an initial model in PGen(T) and hence in PMod(T), as the following example shows.

Let $\Sigma=(S, F)$ be the signature defined by $S=\{s\}, F=\{a, b: \rightarrow s\}$, Ax consist only of the axiom $\mathrm{a}=\mathrm{b} \supset \mathrm{D}(\mathrm{b})$ and $\mathrm{T}_{1}$ be the conditional type $(\Sigma, \mathrm{Ax})$.

We can define two models $A$ and $B$ by:

$$
\mathrm{s}^{\mathrm{A}}=\{1\}, \quad \mathrm{a}^{\mathrm{A}}=1, \mathrm{~b}^{\mathrm{A}} \text { undefined; } \quad \mathrm{s}^{\mathrm{B}}=\mathrm{s}^{\mathrm{A}}, \quad \mathrm{b}^{\mathrm{B}}=1, \mathrm{a}^{\mathrm{B}} \text { undefined. }
$$

Therefore, if $C$ is an algebra s.t. $t^{C} \in s^{C}$ iff $\left(t^{B} \in s^{B}\right.$ for all $\left.B \in P M o d\left(T_{1}\right)\right)$, then ${ }_{a} C$ and $b^{C}$ are undefined; thus $\mathrm{C} \vDash \mathrm{a}=\mathrm{b}$ and $\mathrm{C} \neq \mathrm{D}(\mathrm{b})$, and hence $\mathrm{C}$ is not a model.

2. If $\mathrm{MDef}(\mathrm{PGen}(\mathrm{T}))$ is not empty, then there exists a model I initial in $\mathrm{MDef}(\mathrm{PGen}(\mathrm{T}))$; however in general this model is not initial in $\mathrm{PGen}(\mathrm{T})$, as the following example shows.

Let $\Sigma=(S, F)$ be the signature defined by $S=\{s\}$ and $F=\{a, b, c, d: \rightarrow s\}$, Ax be the set of conditional formulae $\{D(c), D(d), a=b \supset c=d\}$ and $T_{2}$ be the conditional type $(\Sigma, A x)$.

For any minimally defined model $A, a^{A}$ and $b^{A}$ are undefined and thus $A \vDash a=b$. Thus all term-generated and minimally defined models are isomorphic to $I$, defined by:

$$
\mathrm{s}^{\mathrm{I}}=\{1\}, \quad \mathrm{c}^{\mathrm{I}}=1, \mathrm{~d}^{\mathrm{I}}=1, \mathrm{a}^{\mathrm{I}}, \mathrm{b}^{\mathrm{I}} \text { undefined, }
$$

Therefore $I$ is initial in $\operatorname{MDef}\left(\mathrm{PGen}\left(\mathrm{T}_{2}\right)\right)$, but not in $\mathrm{PGen}\left(\mathrm{T}_{2}\right)$, because there exist models $A \in P G e n\left(\mathrm{~T}_{2}\right)$ s.t. $A \neq c=d$.

3. There exist classes PMod(T) admitting an initial model which are not definable by only positive conditional formulae, as the following example shows.

Let $\Sigma$ be the signature $(\{s\},\{a, b, c, d: \rightarrow s\}), A x$ be the singleton set $\{a=b \supset c=d\}, T$ be the type $(\Sigma, A x)$. Then there exists a model $I$ of $T$, which is initial in PMod(T) and is defined by:

$$
\mathrm{s}^{\mathrm{I}}=\varnothing ; \mathrm{a}^{\mathrm{I}}, \mathrm{b}^{\mathrm{I}}, \mathrm{c}^{\mathrm{I}}, \mathrm{d}^{\mathrm{I}} \text { undefined. }
$$

In order to show that there does not exist a positive conditional type PT s.t. $\mathrm{PMod}(\mathrm{PT})=\mathrm{PMod}(\mathrm{T})$, it is sufficient to show that there exists a subclass $C$ of $\operatorname{PMod}(T)$ s.t. $W_{\Sigma} / K C \notin \operatorname{PMod}(T)$, since $\mathrm{W} \Sigma / \mathrm{K}^{\prime} \in \mathrm{PMod}(\mathrm{PT})$ for all positive conditional types PT and all subclass $C^{\prime}$ of PMod(PT), because of prop. 1.3 .

Let $C$ be the class $\{A, B\}$, where $A$ and $B$ are defined by:

$$
\begin{array}{ll}
\mathrm{s}^{\mathrm{A}}=\{\cdot, @\} ; & \mathrm{a}^{\mathrm{A}}=\cdot \cdot \mathrm{b}^{\mathrm{A}} \text { undefined, } \mathrm{c}^{\mathrm{A}}=@, \mathrm{~d}^{\mathrm{A}} \text { undefined; } \\
\mathrm{s}^{\mathrm{B}}=\mathrm{s}^{\mathrm{A}} ; & \mathrm{a}^{\mathrm{B}} \text { undefined, } \mathrm{b}^{\mathrm{B}}=\cdot, \mathrm{c}^{\mathrm{B}}=@, \mathrm{~d}^{\mathrm{B}} \text { undefined, }
\end{array}
$$

Both are models of $T$. Then $W_{\varepsilon} / K^{C}$ is isomorphic to the algebra $C$ defined by:

$$
{ }_{\mathrm{s}} \mathrm{C}=(\cdot), \quad \mathrm{a}_{\mathrm{C}}^{\mathrm{C}}, \mathrm{b}^{\mathrm{C}}, \mathrm{d}^{\mathrm{C}} \text { undefined, } \mathrm{c}^{\mathrm{C}}=\cdot \text {. }
$$

Thus $W_{\Sigma} / K^{C}$ is not a model of $T$ and hence there does not exist a positive conditional type PT s.t. PMod(PT) $=$ PMod(T).

This counterexample could be presented using a different terminology. Note that $\mathrm{s}^{C}=\mathrm{s}^{\mathrm{A}} \cap \mathrm{s}^{\mathrm{B}}$ and hence the class of models of $T$ is not closed w.r.t. the intersection of congruences and so it is not a quasi-variety, contrary to the fact that the classes of models of positive conditional types are quasi-varieties (see [T] for references and for a generalization of this results to arbitrary abstract algebraic institutions).

4. Let us consider again the types $T_{1}$ and $T_{2}$ of remarks 1 and 2 above. The lack of the initial model for $T_{1}$ is due to the particular form of the axiom $a=b \supset D(b)$ : since there exist models where $a$ is undefined and the same happens for $b$, if the initial model $I$ exists, then $a^{I}$ and $b^{I}$ are both undefined and equal, so that the axiom, which would imply $D(b)$, cannot be met. A partly similar situation is shown by $T_{2}$; the combination of the three axioms $D(c), D(d)$ and $a=b \supset c=d$ produces a situation analogous to the one just discussed: if the 
initial model $\mathrm{I}$ exists, then not only $\mathrm{a}^{\mathrm{I}}$ and $\mathrm{b}^{\mathrm{I}}$ would be undefined and equal, but also $\mathrm{c}^{\mathrm{I}}$ and $\mathrm{d}^{\mathrm{I}}$ should be different, otherwise $c^{\mathrm{I}}=\mathrm{d}^{\mathrm{I}}$, being an existential equality, should hold in every model what is patently false; hence the axiom $a=b \supset c=d$, which would imply $c=d$, cannot be met. Note that the axiom $a=b \supset c=d$ in itself does not prevent the existence of an initial model as it is shown in remark 3 . The formula $a=b \supset c=d$ is causing trouble, we call it "naughty", in connection with the other axioms. Now we will try to capture and formalize the notion of "naughty formula" for a specification and to show that the absence of naughty formulae is a necessary and sufficient condition for the existence of the initial model.

Def. 1.5. Let be given a type $T=(\Sigma, A x)$.

- The set SEEq(T) is the set

$\left\{D(t) \mid t \in W_{\Sigma}\right\} \cup\left\{t=t^{\prime} \mid t, t^{\prime} \in W_{\Sigma}\right.$, either $A \vDash D(t) \forall A \in \operatorname{PMod}(T)$ or $\left.A \vDash D(t) \forall A \in P M o d(T)\right\}$

- The set SNF(T), where SNF stands for Semantic Naughty Formulae, consists of all closed conditional formulae $\wedge \Delta \supset \varepsilon$ s.t.

1. $\wedge \Delta \supset \varepsilon$ is $\alpha\left[t_{x} / x \mid x \in \operatorname{Var}(\alpha)\right]$ for some $\alpha \in A x$ and some $t_{x} \in W_{\Sigma}$ s.t. $A \vDash D\left(t_{x}\right)$ $\forall \mathrm{A} \in \operatorname{PMod}(\mathrm{T})$

2. $A \vDash \delta$ for all $\delta \in \Delta \cap S E E q(T)$ and all $A \in \operatorname{PMod}(T)$;

3. $\varepsilon \in \operatorname{SEEq}(T)$ and there exists $A \in \operatorname{PMod}(T)$ s.t. $A \not \varepsilon$.

The notation SEEq stands for Semantic Existential Equations and is justified by the fact that we have recalled in sec. 1, that $D(t)$ also can be expressed as an existential equation $t=e t$.

Theorem 1.6. (Main Theorem 1) For every type $T$, there exists a model initial in PMod(T) iff $\mathrm{SNF}(\mathrm{T})=\varnothing$.

\section{Initiality and logical deduction}

In the following when referring to generic formulae and inference systems we consider formulae and inference systems within an infinitary logic which extends first-order logic by admitting denumerable conjunctions (, disjunctions) and quantification over denumerable sets of variables (see e.g.[K]). However we will show that we can restrict ourselves to consider only conditional formulae.

Def. 2.1. For a conditional type $T=(\Sigma, A x)$, a conditional system $L(T)$, in the following abbreviated to $c$-system, is an inference system $L(T)$ s.t.:

$\operatorname{cs}_{1} \quad L(T) \vdash \alpha$ for all $\alpha \in \mathbf{A x}$;

cs2 the family $\equiv L(\mathrm{~T})=\left\{\equiv L(\mathrm{~T})_{\mathrm{S}}\right\}_{\mathrm{s} \in \mathrm{S}}$, where

$\equiv^{L}(\mathrm{~T})_{\mathrm{s}}=\left\{\left(\mathrm{t}, \mathrm{t}^{\prime}\right) \mid \mathrm{t}, \mathrm{t}^{\prime} \in \mathrm{W}_{\Sigma \mid \mathrm{s}}, L(\mathrm{~T}) \vdash \mathrm{D}(\mathrm{t}), L(\mathrm{~T}) \vdash \mathrm{D}\left(\mathrm{t}^{\prime}\right), L(\mathrm{~T}) \vdash \mathrm{t}=\mathrm{t}^{t}\right\}$,

is a strict congruence over $\mathrm{W}_{\Sigma}$ s.t. $\operatorname{Dom}(\underline{\equiv}(\mathrm{T}))=\left\{\mathrm{t} \mid \mathrm{t} \in \mathrm{W}_{\Sigma}, L(\mathrm{~T}) \vdash \mathrm{D}(\mathrm{t})\right)$;

cs3 for any countable set of elementary formulae $\Delta$, any elementary formula $\eta$, any family $\mathrm{X}$ of variables, and any closed tem $\mathbf{t}_{\mathrm{x}}$ of appropriate sort

$L(\mathrm{~T}) \vdash \wedge \Delta \supset \eta$ and $L(\mathrm{~T}) \vdash \mathrm{D}\left(\mathrm{t}_{\mathrm{X}}\right)$ for all $\mathrm{X} \in \mathrm{X}_{\mathrm{S}}$ and $\mathrm{s} \in \mathrm{S}$ implies $L(T) \vdash \wedge\left\{\delta\left[\left\{\mathrm{t}_{\mathrm{x}} / \mathrm{x} \mid \mathrm{x} \in \mathrm{X}_{\mathrm{S}}, \mathrm{s} \in \mathrm{S}\right\}\right] \mid \delta \in \Delta\right\} \supset \eta\left[\left\{\mathrm{t}_{\mathrm{x}} / \mathrm{x} \mid \mathrm{x} \in \mathrm{X}_{\mathrm{S}}, \mathrm{s} \in \mathrm{S}\right\}\right]$

cs4 for any countable sets of elementary closed formulae $\Theta, \Gamma, \theta_{\gamma}$ and any elementary closed formula $\varepsilon$

$L(T) \vdash \wedge \theta \wedge \wedge \Gamma \supset \varepsilon$ and $L(T) \vdash \wedge \theta_{\gamma} \supset \gamma$ for all $\gamma \in \Gamma$ implies

$L(T) \vdash \wedge \Theta \wedge \wedge\left(U_{\gamma \in \Gamma} \Theta_{\gamma}\right) \supset \varepsilon$

cs5 is sound, ie for any formula $\varphi, L(T) \vdash \varphi$ implies $M \vDash \varphi$ for all $M \in \operatorname{PMod}(T)$.

In order to make the presentation more concrete and to prepare the way to a completeness result, we introduce, for the moment just as an example, a particular c-system, which is reminiscent of systems found in the literature (see, e.g. [WB]); however the peculiar form of the axioms and inference rules will play a very important technical role when dealing with completeness in the next section. 
Def. 2.2. The canonical c-system for a conditional type $T=(\Sigma, A x)$, denoted by $C S(T)$, consists of the axioms $A x$ and of the following axioms:

$$
\begin{aligned}
& 1 \quad t=t \\
& t \in W_{\Sigma i s} \\
& 2 \quad t=t^{\prime} \supset t^{\prime}=t \\
& \mathrm{t}, \mathrm{t}^{\prime} \in \mathrm{W}_{\Sigma \mid s} \\
& 3 \quad t=t^{\prime} \wedge t^{\prime}=t^{\prime \prime} \supset t=t^{\prime \prime} \\
& \mathbf{t}, \mathrm{t}^{\prime}, \mathrm{t}^{\prime \prime} \in \mathrm{W}_{\Sigma \mid \mathrm{s}} \\
& 4 \quad t_{1}=t_{1}^{t} \wedge \ldots \wedge t_{n}=t_{n} \supset o p\left(t_{1}, \ldots, t_{n}\right)=o p\left(t^{t} 1, \ldots, t_{n}\right) \\
& \mathrm{t}_{\mathrm{i}} \in \mathrm{W}_{\Sigma \mathrm{s}_{\mathrm{i}}}, \mathrm{i}=1 \ldots \mathrm{n}, \text { op: } \mathrm{s}_{1} \times \ldots \times s_{\mathrm{n}} \rightarrow s \\
& 5 \quad \mathrm{D}\left(\mathrm{op}\left(\mathrm{t}_{1}, \ldots, \mathrm{t}_{\mathrm{n}}\right)\right) \supset \mathrm{D}\left(\mathrm{t}_{\mathrm{i}}\right) \\
& t_{i} \in W_{\Sigma \mid s_{i}}, i=1 \ldots n, \text { op: } s_{1} \times \ldots \times s_{n} \rightarrow s \\
& 6 D(t) \wedge t=t^{t} \supset D\left(t^{\prime}\right) \\
& \mathfrak{t}, \mathfrak{t}^{\mathfrak{t}} \in \mathrm{W}_{\Sigma \mid \mathrm{s}} \\
& 7 \\
& \frac{\wedge \Theta \wedge \wedge \Gamma \supset \varepsilon,\left\{\wedge \Theta_{\gamma} \supset \gamma \mid \gamma \in \Gamma\right\}}{\wedge \Theta \wedge \wedge\left(\cup_{\gamma \in \Gamma} \Theta_{\gamma}\right) \supset \varepsilon} \\
& 8 \\
& \wedge \Delta \supset \eta \\
& \overline{\wedge\left\{D\left(t_{x}\right) \mid x \in X_{S}, s \in S\right\} \wedge \wedge\left\{\delta\left[\left\{t_{x} / x \mid x \in X_{S}, s \in S\right\}\right] \mid \delta \in \Delta\right\} \supset \eta\left[\left\{t_{x} / x \mid x \in X_{S}, s \in S\right\}\right]}
\end{aligned}
$$

\section{Remarks.}

1 Notice that $\operatorname{CS}(\mathrm{T})$ is really a c-system. First of all it is trivial to verify properties $\mathrm{cs}_{1}, \operatorname{cs}_{2}, \operatorname{cs}_{3}$, cs4. So, in order to show that $C S(T)$ is a $c$-system for $T$, we only have to show that it is sound. Since the soundness of rules 1 to 7 is obvious, we only show the soundness of rule 8 .

Let $A$ be a model of $T, \psi^{*}$ denote, for all formulae $\psi$, the formula $\psi\left[\left\{t_{x} / x \mid x \in X_{S}, S \in S\right\}\right], Y$ be the set $\operatorname{Var}(\wedge \Delta \supset \eta), \mathrm{Y}^{*}$ be $\operatorname{Var}\left(\wedge\left\{\mathrm{D}\left(\mathrm{t}_{\mathrm{X}}\right) \mid \mathrm{X} \in \mathrm{X}\right\} \wedge \wedge\left\{\delta^{*} \mid \delta \in \Delta\right\} \supset \eta^{*}\right)$, ie, since $\mathrm{t}_{\mathrm{x}} \in \mathrm{W}_{\Sigma}$ for all $\mathrm{x} \in \mathrm{X}, \mathrm{Y}^{*}=\operatorname{Var}\left(\wedge\left|\delta^{*}\right| \delta \in \Delta \mid \supset \eta^{*}\right)=\mathrm{Y}-\mathrm{X}$ and $\mathrm{V}$ be a valuation for $\mathrm{Y}^{*}$ in $\mathrm{A}$. Let us assume that $A F_{V} \delta^{*}$ for all $\delta \in \Delta$ and $A \cap V D\left(t_{X}\right)$ for all $x \in X$ and show that $A \vDash V \eta^{*}$. Let $V^{\prime}$ be defined by $V^{\prime}(y)=V(y)$ for all $y \in Y^{*}$ and $V^{\prime}(x)=t_{x}{ }^{A}$ for all $x \in X \cap V \operatorname{ar}(\wedge \Delta \supset \eta)$. First of all we show that $\mathrm{V}^{\prime}$ is a valuation for $\mathrm{Y}$ in $\mathrm{A}$. Since $\mathrm{X} \cap \mathrm{Y}^{*}=\varnothing, \mathrm{V}^{\prime}$ is a (partial) function from $\mathrm{X}$ into $\mathrm{A}$; so we only have to show that it is total. Let $y \in Y$; then either $y \in Y^{*}$, and in this case $V^{\prime}(y)=V(y)$ and so $V^{\prime}(y) \in A$, since $V$ is a valuation, or $y \in X$, and in this case $V^{\prime}(y)=t_{y} A$ and so, since we have assumed $A \vDash V D\left(t_{y}\right), t_{y} A \in A$.

By definition of $V^{\prime}$ and $V$, we also have that $A \vDash V \psi^{*}$ iff $A \vDash^{\prime} \psi$ for all formulae $\psi$ and hence, since we have assumed that $\mathrm{A} \vDash \mathrm{V} \delta^{*}$ for all $\delta \in \Delta$, we also have that $\mathrm{A} \vDash \mathrm{V}^{\prime} \delta$ for all $\delta \in \Delta$; moreover, by inductive hypothesis, $A \vDash V^{*} \wedge \Delta \supset \eta$ and hence $A \vDash V^{*} \eta$ ie $A \vDash V \eta^{*}$.

2. Notice that the variables only may appear in the axioms of $\mathrm{Ax}$ and in rule 8 . Thus in order to eliminate variables from a formula we must apply rule 8 , which disposes of the problems of unsoundness for many-sorted deduction noted by Goguen and Meseguer (see [MG]).

Def. 2.3. For a given conditional type $\mathrm{T}$, a c-system $L(\mathrm{~T})$

- is complete w.r.t. a set $\Theta$ of formulae iff for any $\vartheta \in \Theta$, if $M \vDash \vartheta \forall M \in \operatorname{PMod}(T)$, then $L(T) \vdash \vartheta$.

- is EEq-complete iff it is complete w.r.t. the set SEEq(T); equivalently $L(T)$ is EEq-complete iff it is complete w.r.t the set

$$
\mathrm{EEq}(L(\mathrm{~T}))=\left\{\mathrm{D}(\mathrm{t}) \mid \mathrm{t} \in \mathrm{W}_{\Sigma i \mathrm{~s}}\right\} \cup\left\{\mathrm{t}=\mathrm{t}^{\prime} \mid \mathrm{t}, \mathrm{t}^{\prime} \in \mathrm{W}_{\Sigma \mid \mathrm{s}} \text {, either } L(\mathrm{~T}) \vdash \mathrm{D}(\mathrm{t}) \text { or } L(\mathrm{~T}) \vdash \mathrm{D}\left(\mathrm{t}^{\prime}\right)\right\}
$$

Remark. Notice that for every c-system $L(T)$ completeness w.r.t. $\operatorname{EEq}(L(T))$ and w.r.t. SEEq(T) are really equivalent. Indeed, since every c-system $L(\mathrm{~T})$ is sound, if $L(\mathrm{~T}) \vdash \mathrm{D}(\mathrm{t})$, then $\mathrm{A} \vDash \mathrm{D}(\mathrm{t})$ for all $\mathrm{A} \in \mathrm{PMod}(\mathrm{T})$ 
$\mathrm{A} \in \operatorname{PMod}(\mathrm{T})$ and hence $\operatorname{EEq}(L(\mathrm{~T})) \subseteq \mathrm{SEEq}(\mathrm{T})$. Conversely assume that $L(\mathrm{~T})$ is complete w.r.t. $\mathrm{EEq}(L(\mathrm{~T}))$; thus in particular if $A \vDash D(t)$ for all $A \in P M o d(T)$, then $L(T) \vdash D(t)$ and hence $\left\{t=t^{\prime} \mid t, t^{\prime} \in W_{\Sigma}\right.$, either $A \vDash D(t) \forall A \in \operatorname{PMod}(T)$ or $\left.A \vDash D(t) \forall A \in \operatorname{PMod}(T)\right\} \subseteq$

$$
\left\{\mathrm{t}=\mathrm{t}^{\prime} \mid \mathrm{t}, \mathrm{t}^{\prime} \in \mathrm{W}_{\Sigma \mid s} \text {, either } L(\mathrm{~T}) \vdash \mathrm{D}(\mathrm{t}) \text { or } L(\mathrm{~T}) \vdash \mathrm{D}\left(\mathrm{t}^{\prime}\right)\right\},
$$

ie $\operatorname{SEEq}(T) \subseteq \operatorname{EEq}(L(T))$.

Notice now that, since $\equiv L(T)$ is a congruence because of condition cs2 of def. 2.1, we can define the algebra $\mathrm{W}_{\Sigma}=L(\mathrm{~T})$. Thus we state a proposition which is useful in the following and is a slight generalization of well-known results for total algebras.

Prop. 2.4. The algebra $W_{\Sigma} / L(T)$ is a model of $T$ iff it is initial in $\operatorname{PMod}(T)$.

It is now convenient to give a notion of naughty formula related to a c-system, since it allows us to connect the initial model with logical inference systems.

Def. 2.5. For a given $\mathrm{T}$ and a c-system $L(T)$, the set $\mathrm{NF}(L(T)$ ) (NF for Naughty Formulae) consists of all closed conditional formulae $\varphi$ s.t.

$\mathrm{nf}_{1} \varphi$ is $\alpha\left[\mathrm{t}_{\mathrm{x}} / \mathrm{x} \mid \mathrm{x} \in \operatorname{Var}(\alpha)\right]$ for some $\alpha \in \mathrm{Ax}$ and $\mathrm{t}_{\mathrm{x}} \in \mathrm{W}_{\Sigma}$ s.t. $L(\mathrm{~T}) \vdash \mathrm{D}\left(\mathrm{t}_{\mathrm{x}}\right)$;

$\mathrm{nf}_{2} \quad L(\mathrm{~T}) \vdash \delta$ for all $\delta \in \operatorname{prem}(\varphi) \cap \operatorname{EEq}(L(\mathrm{~T}))$;

$\mathrm{nf}_{3} \quad L(\mathrm{~T}) \nvdash \operatorname{cons}(\varphi)$ and $\operatorname{cons}(\varphi) \in \mathrm{EEq}(L(\mathrm{~T}))$.

Prop. 2.6. For all EEq-complete c-systems $L(T)$ we have $\operatorname{NF}(L(T))=S N F(T)$.

Theorem 2.7. Let $L(\mathrm{~T})$ be a c-system. The set $\mathrm{NF}(L(\mathrm{~T}))$ is empty iff $\mathrm{W}_{\Sigma} \equiv L(\mathrm{~T})$ is a model of $\mathrm{T}$.

Putting together prop. 2.4, prop. 2.6 and theorem 2.7 we get our second main result.

Theorem 2.8.(Main theorem 2 ) Let $\mathrm{T}$ be a conditional type. For every c-system $L(\mathrm{~T})$ the following conditions are equivalent:

1) the set $\mathrm{NF}(L(T))$ is empty;

2) the algebra $W_{\Sigma} \perp$ (T) is a model of $T$;

3) the algebra $W_{\Sigma} \equiv(T)$ is initial in PMod(T).

Moreover, if $L(T)$ is EEq-complete then each one of the above conditions is equivalent to

4) there exists an initial model in PMod(T).

It is easy to obtain the well-known initiality result for positive conditional types [BW1] as a corollary of the above results; for that we state an intermediate result.

Prop. 2.9. Let $\mathrm{T}=(\Sigma, \mathrm{Ax})$ be a conditional type, $L(\mathrm{~T})$ be a c-system and $\mathrm{A}$ be the algebra $\mathrm{W}_{\Sigma}=L(\mathrm{~T})$; then $A \vDash \alpha$ for all positive conditional axioms $\alpha$ of $A x$.

Corollary 2.10 [BW1]. Let $\mathrm{PT}=(\Sigma, \mathrm{Ax})$ be a positive conditional type, $L(\mathrm{PT})$ be a c-system; then the algebra $\mathrm{W}_{\Sigma /}=L(\mathrm{PT})$ is initial in $\mathrm{PMod}(\mathrm{PT})$.

Remark. If we consider a positive conditional type $\mathrm{PT}$, then we have seen that the algebra $\mathrm{W}_{\Sigma} /(\mathrm{PT})$ is the initial model of PT for every c-system $L(\mathrm{PT})$ for PT. However if we consider a conditional type $\mathrm{T}$, even if there exists an initial model in PMod(T), a generic c-system $L(T)$ is too poor for $W_{\Sigma}=(T)$ being the initial model of $T$; for instance, even if there exists an initial model in $\operatorname{PMod}(T), W_{\Sigma} \equiv C S(T)$ is not in general a model of $T$, as the following example shows.

Let $\mathrm{T}_{4}$ be the conditional type $(\Sigma, \mathrm{Ax})$, where $\Sigma$ is the signature $(\{s\},\{\mathrm{a}, \mathrm{b}, \mathrm{c:} \rightarrow \mathrm{s}\})$ and $\mathrm{Ax}$ is the set $\{D(a) \supset a=b, D(b) \supset a=b, a=b \supset D(c)\}$.

Then the formulae $\mathrm{a}=\mathrm{b}$ and $\mathrm{D}(\mathrm{c})$ hold in every model of $\mathrm{T}_{4}$, while $\operatorname{CS}\left(\mathrm{T}_{4}\right) \nvdash \mathrm{a}=\mathrm{b}$ and hence $C S\left(\mathrm{~T}_{4}\right) \nvdash \mathrm{D}(\mathrm{c})$. Moreover there exists an initial model of $\mathrm{T}_{4}$, which is isomorphic to the algebra $\mathrm{A}$ defined by: $\mathrm{s}^{\mathrm{A}}=\{\cdot\}, \mathrm{a}^{\mathrm{A}}, \mathrm{b}^{\mathrm{A}}$ are undefined, $\mathrm{c}^{\mathrm{A}}=\cdot$.

Another interesting consequence of the above results is the following proposition.

Prop. 2.11. Let $\mathrm{T}$ be the conditional type ( $\Sigma, \mathrm{Ax}), L(\mathrm{~T})$ be an EEq-complete c-system and $\mathrm{T}^{+}$be the conditional type $\left(\Sigma, \mathrm{Ax}_{\mathrm{A}} \mathrm{Ax}^{+}\right)$, where $\mathrm{Ax}^{+}$is the set $\mathrm{EForm}(\Sigma, \varnothing)$-SEEq(T). 
1. The following conditions are equivalent:

a1 MDef(PMod(T)) is not empty;

a2 $L(T) \vdash \mathrm{D}(\mathrm{t})$ iff $L\left(\mathrm{~T}^{+}\right) \vdash \mathrm{D}(\mathrm{t})$ for all $\mathrm{t} \in \mathrm{W}_{\Sigma}$;

a3 $W_{\Sigma}{ }^{L\left(T^{+}\right)}$is initial in MDef(PMod(T)).

2. There exists an initial model in PMod(T) iff $\left(L(T) \vdash \varepsilon\right.$ iff $L\left(T^{+}\right) \vdash \varepsilon$ for all $\varepsilon \in$ SEEq(T)).

Remark. We shortly show that the well-known theory of total types, ie of the types whose models are only total algebras, and its results of initiality can be seen as a particular case of partial types. First of all we note that the (total) models of a total type $T T=(\Sigma, A x)$ are exactly the (partial) models of the type $\operatorname{Par}(T T)=(\Sigma, A x \cup A x T o t$, where $\left.A x^{T o t}=\left\{D\left(o p\left(x_{1}, \ldots, x_{n}\right)\right) \mid o p \in F_{(s 1} \ldots s_{n}, s\right)\right\}$ and $x_{i}$ are variables of suitable sort. Moreover if $A$ and $B$ are total algebras, the condition in order to $p=\left\{p_{s}: s^{A} \rightarrow s^{B}\right\}$ be a homomorphism, since op ${ }^{A}\left(a_{1}, \ldots, a_{n}\right)$ is always defined, can be rewritten as follows: $p_{s_{n+1}}\left(o p^{A}\left(a_{1} \ldots a_{n}\right)\right)=o p^{B}\left(p_{s_{1}}\left(a_{1}\right) \ldots p_{s_{n}}\left(a_{n}\right)\right)$; thus every homomorphism between total algebras is really a homomorphism as defined in the theory of total algebras ( see [MG,pg.465]). Therefore the study of the initial model of TT in the framework of total algebras is completely equivalent to the study of initial model of Par(TT) in this framework. Finally note that for every total type TT and every c-system $L\left(\operatorname{Par}(\mathrm{TT})\right.$ ) for $\operatorname{Par}(\mathrm{TT})$, because of $\mathrm{Ax}^{\mathrm{T} T}$, $L(\mathrm{~T}) \vdash \mathrm{D}(\mathrm{t})$ for all closed term $\mathrm{t}$, and hence $N F\left(L(\operatorname{Par}(T T))\right.$ is empty; therefore the algebra $\mathrm{W}_{\Sigma} / L(\operatorname{Par}(T T))$ is the initial model in $\operatorname{PMod}(\operatorname{Par}(T T))$, because of theorem 2.7 .

\section{A complete conditional system}

This section is devoted to the third main result of the paper. We exhibit a complete conditional system and thus, instantiating the second main result, we can say that a necessary and sufficient condition for the existence of the initial model is the absence of formulae which are naughty w.r.t. the exhibited system. A most interesting feature of this system is that it is obtained by adding just one new rule to the canonical conditional system of the previous section. This new rule takes a very intuitive and simple form in the case of axioms with finitary premises, while the generalization to infinitary premises is rather subtle and tricky. So we introduce the basic ideas by discussing a finitary example.

Consider again the simple example $T_{4}$ already seen (remark after corollary 2.10 ). Let $\Sigma$ be the signature ( $\{\mathrm{s}\},\{a, b, c: \rightarrow s\})$, Ax be the set $\{D(a) \supset a=b, D(b) \supset a=b, a=b \supset D(c)\}$ and $T_{4}$ be the conditional type $(\Sigma, A x)$. Then $A \vDash a=b$ for every model $A$ of $T$, since either $a$ and $b$ are both undefined, and hence equal, or at least one of them is defined and hence, because of the axioms, both are defined and equal. Thus we can think of adding to the canonical c-system the following rule

$$
\text { * } \frac{\wedge\left(\Theta_{1} \cup[D(t)\}\right)>\varepsilon, \wedge\left(\Theta_{2} \cup\left\{D\left(t^{\prime}\right)\right\}\right) \supset \varepsilon, \wedge\left(\Theta_{3} \cup\left\{t=t^{\prime}\right\}\right) \supset \varepsilon}{\wedge\left(\Theta_{1} \cup \Theta_{2} \cup \Theta_{3}\right) \supset \varepsilon}
$$

$t, t^{3} \in W_{\Sigma i s}, \theta_{1}, \theta_{2}, \theta_{3}$ are arbitrary finite subsets of $\operatorname{EForm}(\Sigma, \varnothing), \varepsilon \in E F o r m(\Sigma, \varnothing)$.

Rule * holds in first order logic since from the premises we can infer

$$
\wedge\left(\Delta_{1} \cup[D(t)\}\right) \vee \wedge\left(\Delta_{2} \cup\left\{D\left(t^{\prime}\right)\right]\right) \vee \wedge\left(\Delta_{3} \cup\left\{t=t^{\prime}\right]\right) \supset \varepsilon
$$

and thus also $\wedge\left(\Delta_{1} \cup \Delta_{2} \cup \Delta_{3}\right) \wedge\left(\vee\left\{D(t), D\left(t^{1}\right), t=t^{\prime}\right\}\right) \supset \varepsilon$ and finally, since $\vee\left\{D(t), D\left(t^{\prime}\right), t=t^{\prime}\right\}$ is logically valid, $\wedge\left(\Delta_{1} \cup \Delta_{2} \cup \Delta_{3}\right) \supset \varepsilon$. If we add $*$ to rules $1 \ldots 8$ of $C S\left(T_{4}\right)$ then clearly we get *, $C S\left(\mathrm{~T}_{4}\right) \vdash \mathrm{a}=\mathrm{b}$ which we could not get simply in $\operatorname{CS}\left(\mathrm{T}_{4}\right)$.

However, since we want also to handle the extensionality axiom which is intrinsically infinitary, we have to work within infinitary logic and hence we generalize nule * to the case of infinitary premises.

Def. 3.1. The inference system associated with $\mathrm{T}=(\Sigma, \mathrm{Ax})$, denoted $C L(\mathrm{~T})$ (or simply $C L$ when there is no ambiguity ) consists of the axioms and inference rules of $\operatorname{CS}(\mathrm{T})$ and of the following inference rule:

$9 \frac{\left(\wedge \theta_{j} \wedge \wedge \Gamma_{j} \supset \varepsilon \mid j \in J\right)}{\wedge\left(\cup_{j \in J} \Theta_{j}\right) \supset \varepsilon}$

Important Remark. Note that if all the axioms have finitary premises, then rule 9 can be replaced by rule *. subsets of EForm $(\Sigma, \varnothing)$, $\varepsilon \in \operatorname{EForm}(\Sigma, \varnothing)$. $\forall \Psi \in F u l l i n t e r(\Gamma) \exists t, t^{2} \in W_{\Sigma}$ s.t. $D(t), D\left(t^{3}\right), t=t^{\prime} \in \Psi$, where Fullinter $(\Gamma)=\left\{\Psi \mid \Psi \subseteq\left(\cup_{j \in J} \Gamma_{j}\right), \Psi \cap \Gamma_{j} \neq \varnothing, \forall j \in J\right\}$.

$J$ is an arbitrary set (possibly more than countable), $\Theta_{j}, \Gamma_{\mathbf{j}}$ are arbitrary countable 
Prop. 3.2. The inference system $C L(T)$ is a c-system for $T$.

Remark. Notice that rule 9 is clearly a generalization of rule * given above. Now we show an example of use of rule 9 in an infinitary case.

Let $\Sigma$ be the signature $\left(\left\{s_{1}, s_{2}, s_{3}\right\},\left\{a_{j}: \rightarrow s_{j} ; f_{i j}, g_{i}: s_{i} \rightarrow s_{i} \mid i=1,2, j=1,2,3\right\}\right), \quad x_{i}$ belong to $\operatorname{Var}_{s_{i}}$ for $i=1,2, A x$ be the set $\left\{\alpha_{1}, \alpha_{2}, \alpha_{3}\right\}$, where $\alpha_{1}=\left(D\left(x_{1}\right) \wedge D\left(x_{2}\right) \supset D\left(a_{3}\right)\right)$, $\alpha_{i+1}=\left(\wedge\left\{f_{i}^{n}\left(a_{i}\right)=g_{i}{ }^{n}\left(a_{i}\right) \mid n \in \mathbb{N}\right\} \supset D\left(a_{3}\right)\right)$ for $i=1,2$. From $\alpha_{1}$, by rule 7 , we deduce

$\vartheta^{1} 1_{i, j}=\left(D\left(f_{1} i_{(a 1}\right)\right) \wedge D\left(f_{2} j_{\left.\left(a_{2}\right)\right)} \supset D\left(a_{3}\right)\right)$;

$\vartheta^{2} i_{i j}=\left(D\left(f_{1}{ }^{i}\left(a_{1}\right)\right) \wedge D\left(g_{2}{ }^{j}\left(a_{2}\right)\right) \supset D\left(a_{3}\right)\right)$;

$\vartheta^{3}{ }_{i, j}=\left(D\left(g_{1}{ }^{i}\left(a_{1}\right)\right) \wedge D\left(f_{2}{ }^{j}\left(a_{2}\right)\right) \supset D\left(a_{3}\right)\right)$;

$\vartheta^{4}{ }_{i, j}=\left(D\left(g_{1}{ }^{i}\left(a_{1}\right)\right) \wedge D\left(g_{2} j\left(a_{2}\right)\right) \supset D\left(a_{3}\right)\right)$;

for all $i, j \in \mathbb{N}$. Thus from $\alpha_{2}, \alpha_{3}$ and $\left(\vartheta_{\mathrm{i}, j} \mid \mathrm{k}=1, \ldots, 4, \mathrm{i}, \mathrm{j} \in \mathbb{N}\right\}$, by rule 9 , we deduce $D\left(a_{3}\right)$; indeed let $\mathrm{J}$ be the set $\left\{\operatorname{prem}\left(\alpha_{2}\right)\right\} \cup\left\{\operatorname{prem}\left(\alpha_{3}\right)\right\} \cup\left\{\operatorname{prem}\left(\vartheta_{\mathrm{i}, j}\right) \mid \mathrm{k}=1, \ldots, 4 ; \mathrm{i}, \mathrm{j} \in \mathbb{N}\right\}$ and $\Psi$ belong to FullInter $(\{\Gamma \mid \Gamma \in J\})$ then, since, by definition of Fullinter( $\{\Gamma \mid \Gamma \in J\}), \quad\left\{\operatorname{prem}\left(\varepsilon_{2}\right)\right\} \cap \Psi \neq \varnothing$ and $\left\{\operatorname{prem}\left(\varepsilon_{3}\right)\right\} \cap \Psi \neq \varnothing$, there exist $m, n \in \mathbb{N}$ s.t. $\left(f_{1} m_{\left(a_{1}\right)}=g_{1}{ }^{m}\left(a_{1}\right)\right),\left(f_{2}{ }^{n}\left(a_{2}\right)=g_{2}{ }^{n}\left(a_{2}\right)\right) \in \Psi$; moreover, since, by definition of Fullinter $(\{\Gamma \Gamma \in J\})$, prem $\left(\vartheta_{m, n}\right) \cap \Psi \neq \varnothing$ for $k=1, \ldots, 4$, we have that:

$$
\begin{aligned}
& \left\{\mathrm{D}\left(\mathrm{f}_{1} \mathrm{~m}^{\mathrm{m}}\left(\mathrm{a}_{1}\right)\right), \mathrm{D}\left(\mathrm{f}_{2}{ }^{\mathrm{n}}\left(\mathrm{a}_{2}\right)\right)\right\} \cap \Psi \neq \varnothing, \quad\left\{\mathrm{D}\left(\mathrm{f}_{1} \mathrm{~m}^{\mathrm{m}}\left(\mathrm{a}_{1}\right)\right), \mathrm{D}\left(\mathrm{g}_{2}{ }^{\mathrm{n}}\left(\mathrm{a}_{2}\right)\right)\right\} \cap \Psi \neq \varnothing, \\
& \left\{\mathrm{D}\left(\mathrm{g}_{1} \mathrm{~m}^{\mathrm{m}}\left(\mathrm{a}_{1}\right)\right), \mathrm{D}\left(\mathrm{f}_{2}{ }^{\mathrm{n}}\left(\mathrm{a}_{2}\right)\right)\right\} \cap \Psi \neq \varnothing, \quad\left\{\mathrm{D}\left(\mathrm{g}_{1} \mathrm{~m}^{\mathrm{m}}\left(\mathrm{a}_{1}\right)\right), \mathrm{D}\left(\mathrm{g}_{2}{ }^{\mathrm{n}}\left(\mathrm{a}_{2}\right)\right)\right\} \cap \Psi \neq \varnothing ;
\end{aligned}
$$

thus either $\left\{D\left(f_{1}{ }^{m}\left(a_{1}\right)\right), D\left(g 1^{m}\left(a_{1}\right)\right)\right\} \subseteq \Psi$ or $\left\{D\left(f_{2}{ }^{n}\left(a_{2}\right)\right), D\left(g 2^{n}\left(a_{2}\right)\right)\right\} \subseteq \Psi$ and hence either

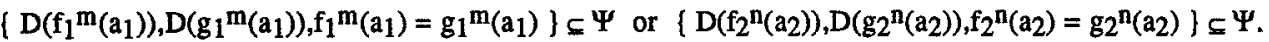

We now proceed to show in outline the relative completeness of $C L(T)$ w.r.t. the closed elementary formulae. We need some definitions and preliminary results (whose proofs are omitted).

Def. 3.3.

- For a given conditional type $T$, the set PNF(T) (for Possibly Naughty Formulae) consists of all closed conditional formulae $\varphi$ s.t.

- $C L(\mathrm{~T}) \vdash \varphi$

- $C L(\mathrm{~T}) b \operatorname{cons}(\varphi)$.

- An $r$-choice (for resolving choice) $\mathrm{C}$ is a set of closed elementary formulae s.t.

for all $\varphi \in \operatorname{PNF}(T)$, if $(\operatorname{prem}(\varphi) \cap \operatorname{EEq}(C L(T))) \subseteq C$, then either $\operatorname{cons}(\varphi) \in \mathrm{C}$, or there exists $\left(t=t^{\prime}\right) \in \operatorname{prem}(\varphi)-\operatorname{EEq}(C L(T))$ s.t. $\left(t=t^{\prime}\right),\left(t^{\prime}=t\right) \notin C$ and either $D(t)$ or $D\left(t^{\prime}\right)$ belongs to $C$.

- The set of all $\mathrm{r}$-choices is denoted by $R$-Choice.

Prop. 3.4. (Deduction Theorem) Let $\mathrm{T}$ be the conditional type $(\Sigma, \mathrm{Ax})$ and $\Gamma$ be a set of elementary closed formulae. Then $C L(\Sigma, A x \cup \Gamma) \vdash \wedge \Theta \supset \varepsilon$ iff $C L(T) \vdash \wedge \Theta \wedge \wedge \Delta \supset \varepsilon$, for an opportune $\Delta \subseteq \Gamma$.

Prop. 3.5. For all conditional types $\mathrm{T}=(\Sigma, \mathrm{Ax})$ and all $\mathrm{r}$-choices $\mathrm{C}$, we have $\mathrm{NF}(C L(\Sigma, \mathrm{Ax} \cup \mathrm{C}))=\varnothing$.

Prop. 3.6. If $T=(\Sigma, A x)$ is a conditional type and $\varepsilon$ is an elementary closed formula s.t. $C L(T) \nvdash \varepsilon$, then there exists an r-choice $\mathrm{C}$ s.t. $C L(\Sigma, \mathrm{Ax} \cup \mathrm{C}) \forall \varepsilon$.

Theorem 3.7.(Main theorem 3 ) The system $C L(T)$ is complete w.r.t. the elementary closed formulae.

Proof. Let $\varepsilon$ be an elementary closed formula and assume that $C L(T) \nvdash \varepsilon$. We divide the proof in two cases.

a Let $\varepsilon$ belong to $\operatorname{EEq}(C L(T))$; we show that there exists a model $A$ of $T=(\Sigma, A x)$ s.t. $A \models \varepsilon$.

- If $\mathrm{NF}\left(C L(\mathrm{~T})\right.$ ) is empty, then $\mathrm{A}=\mathrm{W}_{\Sigma} /{ } C L(\mathrm{~T})$ is a model, because of the theorem 2.7 , and, by construction of $=2 L(\mathrm{~T}), \mathrm{A} \neq \varepsilon$.

- Otherwise there exists an r-choice $C$ s.t. $C L(\Sigma, A x \cup C) \nvdash \varepsilon$, because of prop. 3.6. Moreover $\operatorname{NF}\left(C L\left(T^{\prime \prime}\right)\right)$ is empty, where $T^{\prime \prime}=(\Sigma, A x \cup C)$, because of prop. 3.5; thus $A=W_{\Sigma} / \equiv C L\left(T^{\prime \prime}\right)$ is a model of $T$, because of theorem 2.7. Finally $A$ belongs to $\operatorname{PMod}(T)$, since $\operatorname{PMod}\left(T^{\prime}\right) \subseteq \operatorname{PMod}(T)$ by definition of $T$, and $A \notin \varepsilon$, by definition of $A$.

b Let $\varepsilon$ have the form $\mathrm{t}=\mathrm{t}^{\prime}, C L(\mathrm{~T}) \nvdash \mathrm{D}(\mathrm{t})$, and $C L(\mathrm{~T}) \nvdash \mathrm{D}\left(\mathrm{t}^{\prime}\right)$; if there exists a conditional type $\mathrm{T}^{\prime \prime}$ s.t.

1. $\operatorname{PMod}\left(T^{\prime}\right) \subseteq \operatorname{PMod}(\mathrm{T})$;

2. $\varepsilon \in \operatorname{EEq}\left(C L\left(T^{\prime}\right)\right)$;

3. $C L\left(\mathrm{~T}^{\prime}\right) \not \varepsilon \varepsilon$; 
then, because of a and of conditions 2 and 3, there exists a model A of T" s.t A $\varepsilon$ and hence, because of $1, \mathrm{~A}$ is also a model of $\mathrm{T}$ which does not satisfy $\varepsilon$. Therefore we only have to show that there exists such a $T$. Let $T_{1}$ be the type $(\Sigma, A x \cup\{D(t)\})$ and $T_{2}$ be the type $(\Sigma, A x \cup\{D(t)\})$; we show that either $C L\left(\mathrm{~T}_{1}\right) \forall \mathrm{t}=\mathrm{t}^{t}$ or $C L\left(\mathrm{~T}_{2}\right) \forall \mathrm{t}=\mathrm{t}^{\prime}$. By contradiction we assume that $C L\left(\mathrm{~T}_{1}\right) \vdash \mathrm{t}=\mathrm{t}^{\prime}$ and $C L\left(\mathrm{~T}_{2}\right) \vdash \mathrm{t}=\mathrm{t}^{\prime}$ and prove that $C L(\mathrm{~T}) \vdash \mathrm{t}=\mathrm{t}^{\prime}$. By the absurd hypothesis, because of prop. 3.4, we have $C L(T) \vdash \mathrm{D}(\mathrm{t}) \supset \mathrm{t}=\mathrm{t}^{\prime}$ and $C L(\mathrm{~T}) \vdash \mathrm{D}\left(\mathrm{t}^{\prime}\right) \supset \mathrm{t}=\mathrm{t}^{\prime}$; moreover by rule 2 we have that $C L(\mathrm{~T}) \vdash \mathrm{t}=\mathrm{t}^{\prime} \supset \mathrm{t}^{\mathrm{t}}=\mathrm{t}$ and $C L(\mathrm{~T}) \vdash \mathrm{t}^{\prime}=\mathrm{t} \supset \mathrm{t}=\mathrm{t}^{\mathrm{t}}$ and hence by rule 7 we also have that $C L(T) \vdash t=t^{*} \supset t=t^{*}$. Thus, applying rule 9 to the set $\left\{\mathrm{D}(\mathrm{t}) \supset \mathrm{t}=\mathrm{t}^{\prime}, \mathrm{D}\left(\mathrm{t}^{\prime}\right) \supset \mathrm{t}=\mathrm{t}^{t}, \mathrm{t}=\mathrm{t}^{\prime} \supset \mathrm{t}=\mathrm{t}^{t}\right\}$, we have $C L(T) \vdash t=t^{t}$. Therefore either $C L\left(\mathrm{~T}_{1}\right) \forall \mathrm{t}=\mathrm{t}^{\prime}$, and in this case let $\mathrm{T}^{\prime}$ be $\mathrm{T}_{1}$, or $C L\left(\mathrm{~T}_{2}\right) \forall \mathrm{t}=\mathrm{t}^{\prime}$, and in this case let $\mathrm{T}^{\prime \prime}$ be $\mathrm{T}_{2}$. In any case $\mathrm{T}$ " satisfies conditions $1,2,3$ by definition.

Putting together theorem 2.8 and theorem 3.7 , we get the following conclusive result about initiality.

Theorem 3.8. The following conditions are equivalent:

1. the set $\mathrm{NF}(C L(T))$ is empty;

2. the algebra $\mathrm{W}_{\Sigma} \equiv C L(\mathrm{~T})$ is a model of $\mathrm{T}$;

3. the algebra $\mathrm{W}_{\Sigma}=C L(\mathrm{~T})$ is initial in $\operatorname{PMod}(\mathrm{T})$;

4. there exists a model which is initial in $\operatorname{PMod}(T)$.

\section{Higher-order types}

In this section we apply the results to higher-order specifications. After reducing higher-order specifications to particular classes of first-order specifications (see [MTW]), we consider positive conditional higher-order types, ie higher-order specifications where the only non-positive axioms are the axioms of term-extensionality: two functions are equal iff they coincide over all arguments which are values of terms (clearly term-extensionality coincides with ex-tensionality for term-generated models). As an application of our previous main results, we obtain necessary and sufficient conditions for the existence of initial models of higher-order specifications (theorem 4.5 and corollary 4.7).

Def. 4.1.

- If $S$ is a set, then the set $S \rightarrow$ of functional sorts over $S$ is inductively defined by: $S \subseteq S \rightarrow$ and if $\mathrm{s}_{1}, \ldots, \mathrm{s}_{\mathrm{n}}, \mathrm{s}_{\mathrm{n}+1} \in \mathrm{S}^{\rightarrow}$, then $\mathrm{s}=\left(\mathrm{s}_{1} \times \ldots \times \mathrm{s}_{\mathrm{n}} \rightarrow \mathrm{s}_{\mathrm{n}+1}\right) \in \mathrm{S}^{\rightarrow}$ for all $\mathrm{n} \geq 1$.

- A higher-order signature consists of a set $S$ of basic sorts and of a family $F$ of sets of operation symbols,

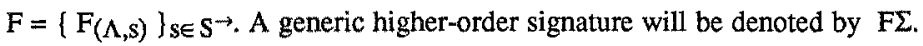

- Let $F \Sigma=(\mathrm{S}, \mathrm{F})$ be a higher-order signature. The associated extended signature is the (first-order) signature defined by $E(F \Sigma)=(S \rightarrow F \cup$ Fapply $)$, where Fapply is the family

$\left\{F^{\text {apply }}\left(\mathrm{s}_{1} \ldots \mathrm{s}_{\mathrm{n}}, \mathrm{s}_{\mathrm{n}+1}\right)\right\}_{\mathrm{n} \geq 1, \mathrm{~s}=\left(\mathrm{s}_{1} \times \ldots \times \mathrm{s}_{\mathrm{n}} \rightarrow \mathrm{s}_{\mathrm{n}+1}\right) \in \mathrm{S} \rightarrow \text {, with Fapply }}\left(\mathrm{ss}_{1} \ldots \mathrm{s}_{\mathrm{n}}, \mathrm{s}_{\mathrm{n}+1}\right)=\left\{\operatorname{apply}_{\mathrm{S}}\right\}$.

We will often use the infix notation for the applys operators, ie we will write $f\left(a_{1}, \ldots, a_{n}\right)$ for apply $y_{s}\left(f, a_{1}, \ldots, a_{n}\right)$, dropping the sort indexes where there is no ambiguity.

- Let $\mathrm{F} \Sigma=(\mathrm{S}, \mathrm{F})$ be a higher-order signature. A higher-order partial algebra $\mathrm{A}$ on $\mathrm{F} \Sigma$ is a partial algebra on $\mathrm{E}(\mathrm{F \Sigma})$ which satisfies the following extensionality condition:

for all $s=\left(s_{1} \times \ldots \times s_{n} \rightarrow s_{n+1}\right) \in S^{\rightarrow}$, with $n \geq 1$ and for all $f, g \in s^{A}$,

if for all $a_{i} \in s i_{i}^{A}, i=1, \ldots, n, f\left(a_{1}, \ldots, a_{n}\right)=g\left(a_{1}, \ldots, a_{n}\right)$, then $f=g$.

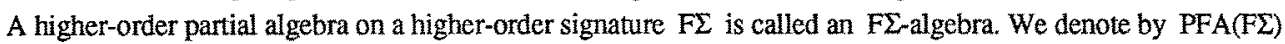
the class of all $\mathrm{F} \Sigma$-algebras.

Remarks. Let PT be a positive (higher-order) conditional type (E(FE),Ax) and Fun-Mod(PT) be the class $\mathrm{PMod}(\mathrm{PT}) \cap \mathrm{PFA}(\mathrm{F} \Sigma)$. 
1 In general there does not exist an $\mathrm{E}(\mathrm{F} 2)$-algebra initial in Fun-Mod(PT), as the following example shows.

Let $\mathrm{PT}$ be the positive type $\left(\mathrm{E}\left(\mathrm{F} \Sigma_{1}\right), \mathrm{Ax} \mathrm{x}_{1}\right)$, where $\mathrm{F} \Sigma_{1}$ is the signature $(\{\mathrm{s}\},\{\mathrm{e}: \rightarrow \mathrm{s} ; \mathrm{f}, \mathrm{g}: \rightarrow(\mathrm{s} \rightarrow \mathrm{s})\})$ and $A x_{1}$ is the set $\{D(f), D(g), D(f) \wedge f=g \supset D(f(e))\}$.

Assume by contradiction that $I$ is initial in Fun-Mod(PT).

Let $F$ and $G$ be the $F \Sigma_{1}$-algebras defined by

$\mathrm{s}^{\mathrm{F}}=\{\cdot\} ;(\mathrm{s} \rightarrow \mathrm{s})^{\mathrm{F}}=\{\varphi, \xi\}$, where $\varphi(\cdot)$ is undefined and $\xi(\bullet)=\cdot ; \mathrm{e}^{\mathrm{F}}=\cdot ; \mathrm{f}^{\mathrm{F}}=\xi, \mathrm{g}^{\mathrm{F}}=\varphi$.

$s^{G}=s^{F} ;(s \rightarrow s)^{G}=(s \rightarrow s)^{F} ; e^{G}=\cdot ; f^{G}=\varphi ; g^{G}=\xi$.

Both $F$ and $G$ belong obviously to Fun-Mod(PT); thus there exist two homomorphisms $p^{F}: I \rightarrow F$ and $p^{G}: I \rightarrow G$.

Since homomorphisms are total functions, for all $a \in s^{I}, p^{F}(a)=\cdot$ and hence $g^{I}(a)$ must be undefined, since $g^{F}(\cdot)$ is undefined and $g^{I}(a) \in s^{I}$ implies, by definition of homomorphism, $\mathrm{p}^{\mathrm{F}}\left(\mathrm{g}^{\mathrm{I}}(\mathrm{a})\right)=\mathrm{e}^{\mathrm{F}}\left(\mathrm{p}^{\mathrm{F}}(\mathrm{a})\right)=\mathrm{e}^{\mathrm{F}}(\cdot)$. Analogously $\mathrm{p}^{\mathrm{G}}(\mathrm{a})=\cdot$ and hence, since $\mathrm{f}^{\mathrm{G}}(\cdot)$ also is undefined, $\mathrm{f}^{\mathrm{I}}(\mathrm{a})$ is undefined too. Thus for all $a \in s^{I}$ we have that both $f^{\mathrm{I}}(a)$ and $g^{\mathrm{I}}(a)$ are undefined and hence $\mathrm{f}^{\mathrm{I}}(\mathrm{a})=\mathrm{g}^{\mathrm{I}}(\mathrm{a})$ for all $a \in s^{I}$, ie $f^{I}=g^{I}$. Therefore $f^{I}=g^{I}$ and, since $f^{I}$ is totally undefined, $(f(e))^{I}$ is undefined, contrary to the assumption that $I$, belonging to Fun-Mod(PT), satisfies the axiom $D(f) \wedge f=g \supset D(f(e))$.

2 In general there does not exist a conditional type $\mathrm{T}$ s.t. $\mathrm{Fun}-\operatorname{Mod}(\mathrm{PT})=\mathrm{PMod}(\mathrm{T})$; indeed, if $\mathrm{A}$ is a higherorder algebra, then in general $W_{E}(F \Sigma) / K^{A}$ is not higher-order (consider for instance the algebras $F$ and $G$ of the example above) while for every conditional type $T=(\Sigma, A x)$ and every model $B$ of $T$ the algebra $\mathrm{W}_{\Sigma} / \mathrm{K}^{\mathrm{B}}$ is a model of $\mathrm{T}$ too.

Therefore we restrict the class of higher-order algebras to the only algebras satisfying a stronger condition of extensionality; this condition is the weakest compatible wih the requirement that, for any PT, the models of PT satisfying this condition are exactly all the partial models of an suitable conditional type $T$.

Def. 4.2. An FE-algebra $A$ is term-extensional iff for any $f, g \in s^{A}$ with $s=\left(s_{1} \times \ldots \times s_{n} \rightarrow s_{n+1}\right)$ and $n \geq 1$,

if for all $t_{i} \in W E(F \Sigma) s_{i}$, with $i=1, \ldots, n, f\left(t_{1} A, \ldots, t_{n} A\right)=g\left(t_{1} A, \ldots, t_{n} A\right)$, then $f=g . \square$

Def. 4.3.

- A (positive) conditional higher-order type (also higher-order specification) $(\mathrm{P}) \mathrm{FT}=(\mathrm{F} \Sigma, \mathrm{Ax})$ consists of a higher-order signature $\mathrm{F} \Sigma$ and a set $\mathrm{Ax}$ of (positive) conditional axioms over $\mathrm{E}(\mathrm{F \Sigma})$.

A generic (positive) higher-order type will be denoted by (P)FT.

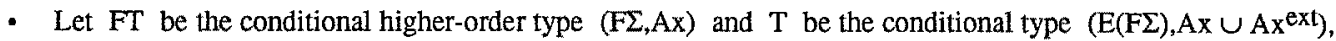
where $\mathrm{Ax}^{\text {ext }}$ is the set

$\left\{\wedge\left(f\left(t_{1}, \ldots, t_{n}\right)=g\left(t_{1}, \ldots, t_{n}\right) \mid t_{i} \in W_{\Sigma \mid s_{i}}, i=1, \ldots, n\right) \supset f=g \mid\left(s_{1} \times \ldots \times s_{n} \rightarrow s_{n+1}\right) \in s^{\rightarrow}\right\}$, and $f, g$ are variables of sort $\left(s_{1} \times \ldots \times s_{n} \rightarrow s_{n+1}\right)$. Then the class FMod(FT) of the higher-order models of $F T$ is defined by FMod(FT) = PMod(T), ie it is the class of all term-extensional models of FT.

Remarks. Although the positive conditional higher-order types are a very special case of conditional types, since they have only one kind of (non-positive) conditional axioms, they have all the limitations of conditional types, as the following examples show.

Let PFT be a positive conditional higher-order type.

1. In general there does not exist an initial model in $\mathrm{Gen}(\mathrm{FMod}(\mathrm{PFT}))$. Indeed, with the notations of the previous remark, let PFT be the higher-order type $\left(F \Sigma_{1}, A x_{1}\right)$. Since $F$ and $G$ are term-generated, then $F$ and $G$ are term-extensional and hence $F, G \in G e n(F M o d(P F T)$ ). Thus, since we have seen that for any algebra $I$ if there exist two homomorphisms $\mathrm{p}^{\mathrm{F}}: \mathrm{I} \rightarrow \mathrm{F}$ and $\mathrm{p}^{\mathrm{G}}: \mathrm{I} \rightarrow \mathrm{G}$, then $\mathrm{I}$ does not satisfy the axiom $D(f) \wedge f=g \supset D(f(e))$, there does not exist an initial model in Gen(FMod(PFT)).

2. Even if there exists a model $\mathrm{I}$ initial in MDef(Gen(FMod(PFT))), in general this model is not initial in Gen(FMod(PFT)).

Let $P F T_{M D}$ be the higher-order type ( $F \Sigma_{M D}, A x_{M D}$ ), where $F \Sigma_{M D}$ is the signature ( $\mathrm{S}_{M D}, F_{M D}$ ), $S_{M D}=\{s\}, \quad F_{M D}=\left(e_{1}, e_{2}: \rightarrow s ; f, g: \rightarrow(s \rightarrow s)\right\}$ and AxMD is the set $\left\{D\left(e_{1}\right), D\left(e_{2}\right), D(f), D(g), D(f) \wedge f=g \supset e_{1}=e_{2}\right\}$. 
For any minimally defined higher-order model $A$ and any closed term $t, f(t)$ and $g(t)$ are undefined; thus $A \vDash f=g$. Therefore every minimally defined model is isomorphic to $I$, defined by:

$s^{\mathrm{I}}=\{1\}, \quad(s \rightarrow s)^{\mathrm{I}}=\{\varphi\}$, where $\varphi(1)$ is undefined, $\mathrm{e}_{1}^{\mathrm{I}}=1, \quad \mathrm{e}_{2}^{\mathrm{I}}=1, \mathrm{f}^{\mathrm{I}}=\varphi, \mathrm{g}^{\mathrm{I}}=\varphi$, which is initial in $\operatorname{MDef}\left(\operatorname{Gen}\left(\operatorname{Mod}\left(\mathrm{PFT}_{\mathrm{MD}}\right)\right)\right)$, but not in $\operatorname{Gen}\left(\operatorname{Mod}\left(\mathrm{PFT}_{\mathrm{MD}}\right)\right)$, because there exist models $A$ s.t. $\quad A \not \mathrm{f}=\mathrm{g}$ and so $\mathrm{A} \not \mathrm{e}_{1}=\mathrm{e}_{2}$.

Thus we explore the existence of initial models for positive conditional higher-order types.

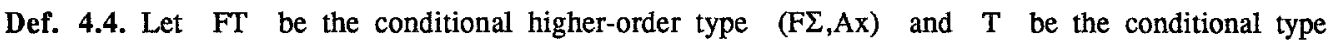
$\left(\mathrm{E}(\mathrm{F \Sigma}), \mathrm{Ax} \cup \mathrm{Ax}{ }^{\mathrm{ext}}\right)$.

- A $c$-system for FT is a c-system for T. In the following a generic c-system will be denoted by $F L(\mathrm{FT})$.

- The system $F C L(\mathrm{FT})$ is the system $C L(T)$.

Theorem 4.5(Main theorem 4). Let PFT be a positive conditional higher-order type and $F L(\mathrm{PFT})$ be a c-system for PFT. The following conditions are equivalent.

1. The algebra $\mathrm{W}_{\mathrm{E}(\mathrm{F \Sigma})}=F L(\mathrm{PFT})$ is initial in $\mathrm{FMod}(\mathrm{PFT})$.

2. For all $\mathrm{f}, \mathrm{g} \in \mathrm{W}_{\mathrm{E}(\mathrm{F} \Sigma) !\left(\mathrm{s}_{1} \times \ldots \times \mathrm{s}_{\mathrm{n}} \rightarrow \mathrm{s}_{\mathrm{n}+1}\right)}, \mathrm{n} \geq 1$, s.t. $F L(\mathrm{PFT}) \vdash \mathrm{D}(\mathrm{f})$ and $F L(\mathrm{PFT}) \vdash \mathrm{D}(\mathrm{g})$ either

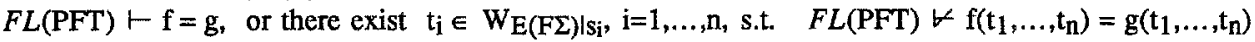
and (either $F L(\mathrm{PFT}) \vdash \mathrm{D}\left(\mathrm{f}\left(\mathrm{t}_{1}, \ldots, \mathrm{t}_{\mathrm{n}}\right)\right)$ or $\left.F L(\mathrm{PFT}) \vdash \mathrm{D}\left(\mathrm{g}\left(\mathrm{t}_{1}, \ldots, \mathrm{t}_{\mathrm{n}}\right)\right)\right)$.

Moreover if $F L(P F T)$ is EEq-complete, then the conditions above are also equivalent to the following:

3) there exists a model of PFT which is initial in FMod(PFT).

Since we know that $F C L(\mathrm{PFT})$ is complete we can instantiate theorem 4.5

Prop. 4.6. Let PFT be a positive conditional higher-order type. The following conditions are equivalent.

1) for all $\mathrm{f}, \mathrm{g} \in \mathrm{W}_{\mathrm{E}}(\mathrm{F \Sigma}) \mid\left(\mathrm{s}_{1} \times \ldots \times \mathrm{s}_{\mathrm{n}} \rightarrow \mathrm{s}_{\mathrm{n}+1}\right), \mathrm{n} \geq 1$, s.t. $F C L(\mathrm{PFT}) \vdash \mathrm{D}(\mathrm{f})$ and $F C L(\mathrm{PFT}) \vdash \mathrm{D}(\mathrm{g})$ either $F C L(\mathrm{PFT}) \vdash \mathrm{f}=\mathrm{g}$, or there exist $\mathrm{t}_{\mathrm{i}} \in \mathrm{WE}(\mathrm{FE}) ! \mathrm{si}, \mathrm{i}=1, \ldots, \mathrm{n}$, s.t. $F C L(\mathrm{PFT}) \vee \mathrm{f}\left(\mathrm{t}_{1}, \ldots, \mathrm{t}_{\mathrm{n}}\right)=\mathrm{g}\left(\mathrm{t}_{1}, \ldots, \mathrm{t}_{\mathrm{n}}\right)$ and (either $F C L(\mathrm{PFT}) \vdash \mathrm{D}\left(\mathrm{f}\left(\mathrm{t}_{1}, \ldots, \mathrm{t}_{\mathrm{n}}\right)\right)$ or $\left.F C L(\mathrm{PFT}) \vdash \mathrm{D}\left(\mathrm{g}\left(\mathrm{t}_{1}, \ldots, \mathrm{t}_{\mathrm{n}}\right)\right)\right)$;

2) the algebra $\mathrm{W}_{\mathrm{E}(\mathrm{F \Sigma})}=F C L(\mathrm{PFT})$ is initial in FMod(PFT);

3) there exists a model of PFT which is initial in FMod(PFT).

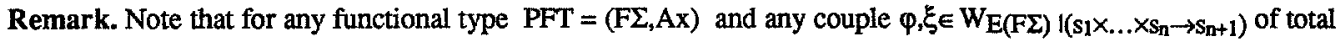
functions, ie of functions s.t. $F C L(\mathrm{PFT}) \vdash \mathrm{D}\left(\varphi\left(\mathrm{x}_{1}, \ldots, \mathrm{x}_{\mathrm{n}}\right)\right)$ and $\left.F C L(\mathrm{PFT}) \vdash \mathrm{D}\left(\xi\left(\mathrm{x}_{1}, \ldots, \mathrm{x}_{\mathrm{n}}\right)\right)\right)$, the condition 1 of prop. 4.6 is satisfied, since for all $t_{i} \in W_{E}(F \Sigma) s_{i}, i=1, \ldots, n, F C L(P F T) \vdash D\left(\varphi\left(t_{1}, \ldots, t_{n}\right)\right)$ and $F C L(\mathrm{PFT}) \vdash \mathrm{D}\left(\xi\left(\mathrm{t}_{1}, \ldots, \mathrm{t}_{\mathrm{n}}\right)\right)$. Thus, in particular, if we want to give a specification for a set of total functions, then we have that condition 1 of prop. 4.6 holds for all $f, g \in W E(F \Sigma) \mid\left(s_{1} \times \ldots \times s_{n} \rightarrow s_{n+1}\right), n \geq 1$, s.t. $F C L(\mathrm{PFT}) \vdash \mathrm{D}(\mathrm{f})$ and $F C L(\mathrm{PFT}) \vdash \mathrm{D}(\mathrm{g})$ and hence there exists an initial model of the specification.

Acknowledgements. We wish to thank Y.Gurevich for a long and helpful discussion of the content of this paper and M.Borga for some useful information on related mathematical logic issues.

\section{References}

[AC1] Astesiano, E.; Cerioli, M. "Free objects and equational deduction for partial (higher-order) conditional specifications", (Technical report, February 1989).

[AR1] Astesiano, E.; Reggio, G. "SMoLCS-Driven Concurrent Calculi", (invited paper) Proc. TAPSOFT 87, vol.1, Berlin, Springer Verlag, 1987 (Lecture Notes in Computer Science n. 249), pp. 169-201.

[AR2] Astesiano, E.; Reggio, G. "An Outline of the SMoLCS Methodology", (invited paper) Mathematical Models for the Semantics of Parallelism, Proc. Advanced School on Mathematical Models of Parallelism (Venturini Zilli, M. ed.), Berlin, Springer Verlag, 1987 (Lecture Notes in Computer Science n. 280), pp. 81-113.

[B] Burmeister, P. A Model Theoretic Oriented Approach to Partial Algebras, Berlin, Akademie-Verlag, 1986, pp. 1-319.

[BW1] Broy, M.; Wirsing, M. "Partial abstract types", Acta Informatica 18 (1982), 47-64. 
[BW2] Broy, M.; Wirsing, M. "On the algebraic specification of finitary infinite communicating sequential processes", Proc. IFIP TC2 Working Conference on "Formal Description of Programming Concepts II", Garmisch 1982.

[K] Keisler, H.J. Model Theory for Infinitary Logic, Amsterdam - London, North-Holland Publishing Company, 1971, pp. 1-208.

[M] Moller, B. "Algebraic Specification with Higher-Order Operations", Proc. IFIP TC 2 Working Conference on Program Specification and Transformation, Bad Tolz F.R.G. 1986 (Meertens, L.G.L.T. ed.), Amsterdam-New York-Oxford-Tokyo, North-Holland Publ. Company, 1987.

[MG] Meseguer, J.; Goguen, J.A. "Initiality, Induction and Computability", Algebraic Methods in Semantics, Cambridge, edited by M.Nivat and J.Reynolds, Cambridge University Press, 1985, pp.459-540.

[MTW] Möller B., Tarlecki A., Wirsing M. “Algebraic Specification with Built-in Domain Constructions", Proceeding of CAAP ' 88 (Nancy France, March 1988), edited by Dauchet M. and Nivat M., Berlin, Springer-Verlag, 1988, pp. 132-148.

[R] Reichel H. Initial Computability, Algebraic Specifications, and Partial Algebras, Berlin (D.D.R.), Akademie-Verlag, 1986.

[T] Tarlecki A. "Quasi-varieties in Abstract Algebraic Institutions", Joumal of Computer and System Science, n. 33 (1986), pp. 333 - 360.

[WB] Wirsing, M.; Broy, M. An analysis of semantic models for algebraic specifications, Intemational Summer School Theoretical Foundation of Programming Methodology, Munich. Germany 28/7 -9/8, 1981.

\section{Appendix: Basic definitions and results on partial algebras}

We start with a short collection of basic notions and results, which are well known. However we need to report them here, in order to fix the notation and also because sometimes there are subtle differences; for example, the notion of congruence differs from the one in [B]. The notation here coincides more or less with that used by Goguen and Meseguer [MG] and Broy and Wirsing [BW1].

Proofs are omitted since they are straightforward adaptation of well known proofs for total algebras (see anyway [B], [MG]).

In the following the symbol = will always denote strong equality, ie if $p$ and $q$ are expressions in the metalanguage, then $p=q$ holds iff either both $p$ and $q$ are undefined, or both are defined and equal. Moreover the word "family" will stay in general for "indexed family", where the indexes are clear from the context.

A signature $(S, F)$ consists of a countable set $S$ of sorts and of a family $F=\left(F_{W}\right)_{w \in S^{*} \times S}$ of sets of operation symbols. We also write op: $s 1 \times \ldots \times s_{n} \rightarrow s$ for $o p \in F\left(s_{1} \ldots s_{n}, s\right)$. A generic signature will be denoted by $\Sigma$.

A partial algebra $A$ on a signature $\Sigma=(\mathrm{S}, \mathrm{F})$ consists of a family $\left\{\mathrm{s}^{\mathrm{A}}\right\}_{\mathrm{S} \in \mathrm{S}}$ of sets, the carriers, and of a family $\left\{o p^{A}\right\}_{o p \in F_{w}, w \in S^{*} \times S}$ of partial functions, the interpretations of operation symbols, $s . t$. if $w=(\Lambda, s)$, with $s \in S$, then either op $A$ is undefined or op ${ }^{A} \in s^{A}$, and if $w=\left(s_{1} \ldots s_{n}, s_{n+1}\right)$, where $n \geq 1$, then op $A: s_{1} A \times \ldots \times s_{n}{ }^{A} \rightarrow s_{n+1} A$. Often we denote the partial algebra $A$ by the couple $\left(\left\{s^{A}\right),\left(o p^{A}\right\}\right)$, omitting the quantifications about $s$ and op which are associated with the signature. A partial algebra over a signature $\Sigma$ is called a $\Sigma$-algebra. We denote by $P A(\Sigma)$ the class of all $\Sigma$-algebras.

A particular example of algebra is the term-algebra, defined in the usual way; in the following we will denote the term-algebra over a signature $\Sigma$ and a family $X$ of variables by $W_{\Sigma}(X)$, or shortly $W_{\Sigma}$ if $X$ is the empty set, and $W_{\Sigma}(\mathrm{X})_{\mid \mathrm{s}}$ will be called the set of the terms of sort $\mathrm{s}$.

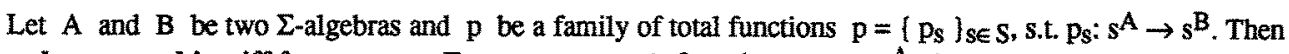
$p$ is a homomorphism iff for any $o p \in F\left(s_{1} \ldots s_{n}, s_{n+1}\right), n \geq 0$, and any $a_{i} \in s_{i} A, i=1, \ldots, n$,

$o p^{A}\left(a_{1}, \ldots, a_{n}\right) \in s_{n+1} A$ implies $p_{s_{n+1}}\left(o p^{A}\left(a_{1}, \ldots, a_{n}\right)\right)=o p^{B}\left(p_{s_{1}}\left(a_{1}\right), \ldots, p_{s_{n}}\left(a_{n}\right)\right)$.

Note that the homomorphisms are composable and that the identity is always a homomorphism; thus we can define a category $\mathbf{C}$ having $\Sigma$-algebras as its objects and homomorphisms as its morphisms; composition and identity are composition and identity as maps.

Two algebras $A$ and $B$ are isomorphic iff there exist two homomorphisms $\left\{\mathrm{p}_{\mathrm{S}}\right\}_{\mathrm{S} \in \mathrm{S}}$ from $\mathrm{A}$ into $\mathrm{B}$ and

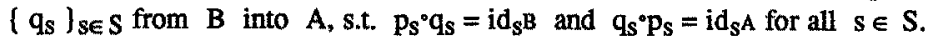


Let $A$ be a $\Sigma$-algebra, $X=\left\{X_{S}\right\}_{S \in S}$ be a family of $S$-sorted variables and $V=\left\{V_{S}: X_{S} \rightarrow s^{A}\right\}_{S \in S}$, be a family of total functions, called a valuation for $\mathrm{X}$ in $\mathrm{A}$. Then the natural interpretation of terms w.r.t. $A$ and $V$, denoted by eval $A, V$, is defined by the following clauses, where we write $t^{A}, V$ for eval $A, V(t)$ :

- $x^{A, V}=V_{S}(x)$, for all $x \in X_{S} ;$ op $A, V=o p^{A}$, for all op $\in F_{W}$, with $w=(\Lambda, s)$;

- $\left(o p\left(t_{1}, \ldots, t_{n}\right)\right)^{A, V}=o p^{A}\left(t_{1} A, V, \ldots, t_{n} A, V\right)$ for all op $\in F\left(s_{1} \ldots s_{n}, s_{n+1}\right), n \geq 1$, and all $\left.t_{i} \in W_{\Sigma}(X)\right|_{s_{j}}$.

When restricted to $W_{\Sigma}$, eval $A, V$ is denoted by eval $A$ and, correspondingly, $t^{A}, V$ becomes $t^{A}$. If eval $A$ is surjective, then $A$ is called term-generated. Note that it is easy to show, by structural induction, that if $A$ and $B$ are two $\Sigma$-algebras and $\mathrm{p}: \mathrm{A} \rightarrow \mathrm{B}$ is a homomorphism, then $\mathrm{p}\left(\mathrm{t}^{\mathrm{A}}\right)=\mathrm{t}^{\mathrm{B}}$ for all closed terms $t \in \mathrm{W}_{\Sigma \mid s}$ s.t. $\mathrm{t}^{\mathrm{A}} \in \mathrm{s}^{\mathrm{A}}$ and hence if $\mathrm{A}$ is term-generated there exists at most one homomorphism from $\mathrm{A}$ into $\mathrm{B}$.

Let $A$ be a $\Sigma$-algebra. The kernel of the natural interpretation of closed terms, denoted by $K^{A}$, is the family $\left\{K^{A_{s}}\right\}_{s \in S}$, where $K^{A}{ }_{s}=\left\{\left(t, t^{\prime}\right) \mid t, t^{\prime} \in W_{\Sigma \mid s}, t^{A}, t^{\prime} A \in s^{A}\right.$ and $\left.t^{A}=t^{\prime} A\right\}$.

Given a signature $\Sigma=(F, S)$ and a $\Sigma$-algebra $A$, a congruence $\equiv$ over $A$ is a family of binary relations $\left\{\equiv_{\mathrm{S}}\right\}_{\mathrm{s} \in \mathrm{S}}$ satisfying the following conditions (where we omit the obvious quantifications over sorts):

C1) $\equiv_{S} \subseteq s^{A} \times S A$, and $\equiv_{S}$ is symmetric, transitive and relatively reflexive, ie if $\left(a, a^{\prime}\right) \in \mathbf{m}_{S}$,

then $(a, a) \in \equiv_{S}$; in the following we denote by $\operatorname{Dom}\left(\equiv_{\mathrm{S}}\right)$ the set $\left\{a \mid(a, a) \in \equiv_{s}\right\}$ and we define $a \equiv D_{s} a^{\prime}$ iff either $a \equiv_{S} a^{\prime}$ or $a, a^{\prime} \notin \operatorname{Dom}\left(\equiv_{S}\right)$;

C2) for any $w=\left(s_{1} \ldots s_{n}, s_{n+1}\right)$, op $\in F_{w}$ and $a_{i}, a_{i}{ }^{\prime} \in s_{i}{ }^{A}, i=1 \ldots n$,

if $a \equiv_{s_{i}} a_{i}^{\prime}$ for $i=1 \ldots n$, then op $A\left(a_{1}, \ldots, a_{n}\right) \equiv D_{s_{n+1}}$ op $A^{A}\left(a_{1}^{\prime}, \ldots, a_{n}^{\prime}\right)$.

A congruence is strict if it also satisfies

C3) for any $o p \in F_{w}$, with $w=\left(s_{1} \ldots s_{n}, s_{n+1}\right)$ and $n \geq 1$, and any $a_{i} \in s_{i} A$ for $i=1, \ldots, n$, if $\operatorname{op}^{A}\left(a_{1}, \ldots, a_{n}\right) \in \operatorname{Dom}\left(\equiv_{S_{n+1}}\right)$, then $a_{i} \in \operatorname{Dom}\left(\equiv_{s_{i}}\right)$, for $i=1, \ldots, n$.

Let $\equiv$ be a congruence over a $\Sigma$-algebra $A$; let [a] denote the equivalence class of a in $\equiv_{S}$ for all $s \in S$ and all $\mathrm{a} \in \mathrm{s}^{\mathrm{A}}$. The quotient algebra of $\mathrm{A}$ w.r.t. $\equiv$, denoted by $\mathrm{A} / \equiv$, is defined by:

- $s^{A} / \equiv=\left\{[a] \mid a \in \operatorname{Dom}\left(\equiv_{s}\right)\right\}$, for all $s \in S$;

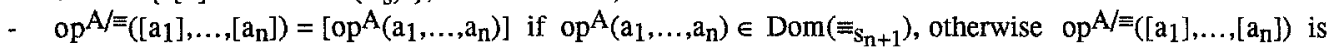
undefined, for all $o p \in F\left(s_{1} \ldots s_{n}, s_{n+1}\right), a_{i} \in \operatorname{Dom}\left(\equiv_{s_{i}}\right), i=1 \ldots n$.

For every family $\equiv=\left\{\equiv^{i}\right\}_{i \in I}$ of congruences over a $\Sigma$-algebra $A$ we denote by $\left.\cap \Leftrightarrow\right)$ the family $\left\{\cap_{\mathrm{i} \in \mathrm{I}} \equiv_{\mathrm{S}}\right\}_{\mathrm{S} \in \mathrm{S}}$. Notice that for all families $\equiv=\left\{\equiv^{\mathrm{i}}\right\}_{\mathrm{i} \in \mathrm{I}}$ of (strict) congruences over a $\Sigma$-algebra $\mathrm{A}$ the family $\cap(\Leftrightarrow)$ is a (strict) congruence over $A$, as it is easy to check.

Notice also that if $\equiv$ is a strict congruence, then for every term $t \in W_{\Sigma}(X)_{\text {s }}$ and every valuation $V$ for $X$ in $A / \equiv$ we have that $\left[t^{A}, V^{\prime}\right]=t^{A}=\equiv, V$, where $V^{\prime}$ is a valuation for $X$ in $A$ s.t. $\left[V^{\prime}(x)\right]=V(x)$, as it easy to show by structural induction. Thus if $A$ is term-generated, then also $A / \equiv$ is term-generated.

Let $C$ be a non-empty subclass of $\mathrm{PA}(\Sigma)$.

- $\operatorname{MDef}(C)$ is the subclass of $C$ defined by:

$\left\{\mathrm{A} \mid \mathrm{A} \in C\right.$ s.t. for all $\mathrm{s} \in \mathrm{S}$, for all $\mathrm{t} \in \mathrm{W}_{\Sigma \mid \mathrm{s}}$, if $\mathrm{t}^{\mathrm{A}} \in \mathrm{s}^{\mathrm{A}}$, then $\mathrm{t}^{\mathrm{B}} \in \mathrm{s}^{\mathrm{B}} \forall \mathrm{B} \in C$ \}.

- $\operatorname{Gen}(C)$ is the subclass of $C$ defined by: $\{\mathrm{A} \mid \mathrm{A} \in C$ s.t. $\mathrm{A}$ is term-generated \}.

- $\mathrm{K}^{C}$ is the congruence defined by $\cap\left(\left\{\mathrm{K}^{\mathrm{A}} \mid \mathrm{A} \in C\right\}\right)$.

- A $\Sigma$-algebra $\mathrm{I}$ is initial in $C$ iff $\mathrm{I} \in C$ and $\forall \mathrm{B} \in C \exists$ a unique homomorphism from $\mathrm{I}$ into $\mathrm{B}$.

Now we can state some results on the existence and the characterization of the initial model for a class $C$ of algebras.

Prop. A. Let $C$ be a non-empty subclass of $\mathrm{PA}(\Sigma)$.

(1) If $\mathrm{I}$ is initial in $C$, then $\mathrm{K}^{\mathrm{I}}=\mathrm{K}^{C}$, ie for all $\mathrm{s} \in \mathrm{S}$

(i1) $\forall t \in W_{\Sigma \mid s}, t^{I} \in s^{I}$ iff $\left(t^{B} \in s^{B}\right.$ for all $\left.B \in C\right)$, or, equivalently, $I \in \operatorname{MDef}(C)$.

(i2) $\forall \mathrm{t}, \mathrm{t}^{\prime} \in \mathrm{W}_{\Sigma \mathrm{s}}$ s.t. $\mathrm{t}^{\mathrm{I}}, \mathrm{t}^{\mathrm{I}} \in \mathrm{s}^{\mathrm{I}}, \mathrm{t}^{\mathrm{I}}=\mathrm{t}^{\mathrm{I}}$ iff $\left(\mathrm{t}^{\mathrm{B}}=\mathrm{t}^{\mathrm{B}}\right.$ for all $\left.\mathrm{B} \in C\right)$.

(2) If there exists an algebra $I \in C$ isomorphic to $\mathrm{W}_{\Sigma} / \mathrm{K}^{C}$, then it is initial in $C$.

(3) If for all $\mathrm{A} \in C$ there exists $\mathrm{B} \in C$ isomorphic to $\mathrm{W}_{\Sigma} / \mathrm{K}^{\mathrm{A}}$, then the following conditions are equivalent

(a) I is initial in $C$;

(b) $I$ is initial in $\operatorname{Gen}(C)$;

(c) $\mathrm{I}$ is isomorphic to $\mathrm{W}_{\Sigma} / \mathrm{K}^{C}$ and $\mathrm{I} \in C$. 